\title{
Walter Eucken als Klassiker der Ordnungsethik - Eine ordonomische Rekonstruktion ${ }^{*}$
}

\section{INGO PIES $^{* *}$}

\section{Walter Eucken as a classical contributor to the ethics of constitutional order - An ordonomic interpretation}

The main proposition of this article is that Walter Eucken can be interpreted as an early and seminal contributor - indeed as a classic - to the ethics of constitutional order. The ordonomic reconstruction proceeds in five steps. The first step identifies several difficulties that tend to impede an appropriate understanding of his works. The second step explains Eucken's problem, while the third step reconstructs his theoretical solution. The fourth step focuses on Eucken as a classical contributor to the ethics of constitutional order. The fifth step finally shows that it is both necessary and possible to further develop the analytical approach pioneered by Walter Eucken.

Keywords: ethics of constitutional order, incentive effects, institutional framework conditions, semantics, social structure, orthogonal position, politics of constitutional order

„[M]it dem vollen Verstehen des Problems ist ein wesentlicher Teil der Lösung bereits vollzogen. “1

Walter Eucken $(* 1891 ;$ †1950) ist der Klassiker eines Denkens in Ordnungen. Hier wird sein Werk aus der Perspektive des ordonomischen Forschungsprogramms betrachtet, das auf Wechselwirkungen zwischen Sozialstruktur und Semantik fokussiert, also auf Interdependenzen zwischen Institutionen und Ideen. Dabei wird deutlich, dass seiner Konzeption von Ordnungspolitik enorme Theorieleistungen - gerade auch im Bereich der Ordnungsethik - zugrunde liegen, die im gegenwärtigen Wissenschaftsbetrieb tendenziell (und zunehmend) unterschätzt werden. ${ }^{2}$

Beitrag eingereicht am 21.02.2011, nach doppelt verdecktem Gutachterverfahren überarbeitete Fassung angenommen am 14.09.2011.

** Prof. Dr. Ingo Pies, Lehrstuhl für Wirtschaftsethik, Martin-Luther-Universität Halle-Wittenberg, Große Steinstraße 73, 06108 Halle (Saale), Tel.: +49 (0)345-55-23421, Fax: +49 (0)345-55-27385, E-Mail: Ingo.Pies@wiwi.uni-halle.de, Forschungsschwerpunkte: Wirtschafts- und Unternehmensethik, Normative Institutionenökonomik, Wissenschaftliche Politikberatung, Global Governance, Corporate Citizenship.

Für kritische Anmerkungen und konstruktive Hinweise dankt der Autor Markus Beckmann, Tobias Braun, Anna König, Stefan Hielscher, Tatjana Schönwälder-Kuntze, Matthias Will sowie zwei anonymen Gutachtern.

$1 \quad$ Eucken (1952/1990: 155).

2 Die folgenden Ausführungen stützen sich zum einen auf das Forschungsprogramm der Ordonomik - vgl. Pies (2009a) und (2009b) -, zum anderen auf ausführliche Untersuchungen 
Die These, dass man von Eucken als einem Klassiker der Ordnungsethik auch heute noch wichtige Lektionen lernen kann, wird in fünf Schritten entwickelt. Der erste Argumentationsschritt identifiziert einige Rezeptionsschwierigkeiten, die einem angemessenen Verständnis seines Gesamtwerks im Wege stehen. Der zweite Schritt erläutert Euckens Problemstellung. Der dritte Schritt rekonstruiert Euckens ordnungspolitischen Denkansatz zur Lösung dieses Problems. Der vierte Schritt richtet den Blick auf die spezifisch ordnungsethische Fundierung der Euckenschen Ordnungspolitik. Im fünften Schritt schließlich wird Eucken als Klassiker ernstgenommen, indem gezeigt wird, dass es nötig - und möglich! - ist, mit Eucken gegen Eucken zu denken, um auf diesem Wege sein Denken in Ordnungen durch ein Denken in Anreizen nachhaltig zu aktualisieren.

\section{Rezeptionsschwierigkeiten}

„Mein Vater erkannte die Gefährdung der modernen Welt. Er sah, dass seine Epoche die wesentlichen Maßstäbe verloren hatte. Er spürte die Erde beben - in einer Zeit, in welcher die meisten Menschen glaubten, sie stünde fester als je.“33

Es gibt subjektive und objektive Faktoren, die einem angemessenen Verständnis des Eucken'schen Gesamtwerks im Wege stehen und dazu führen, dass seine enormen Theorieleistungen in der einschlägigen Sekundärliteratur tendenziell unterschätzt werden. Subjektiv sind solche Rezeptionsschwierigkeiten, die in der Person Walter Euckens und den Besonderheiten seines wissenschaftlichen Gesamtwerks angelegt sind, während hier als objektiv solche Rezeptionsschwierigkeiten bezeichnet werden, die eher in den Eigenheiten des heutigen Wissenschaftsbetriebs begründet sind.

(1) Zu den subjektiven Faktoren gehört, dass Walter Eucken von Hause aus mit einem enormen Bildungshintergrund ausgestattet war. Als Sohn des Philosophen - und Literaturnobelpreisträgers - Rudolf Eucken war Walter Eucken eine gründliche Kenntnis der Schriften Kants und Hegels mitgegeben worden sowie eine Vertrautheit mit der zeitgenössischen Philosophie Edmund Husserls, zu dem auch eine persönliche Bekanntschaft bestand. Walter Eucken ist in der Lebensphilosophie seines Vaters sozialisiert worden und hat seine eigene Problemstellung - durch einen grundlegenden Perspektivwechsel von der Lebensordnung zur Wirtschaftsordnung - aus dieser Philosophie heraus entwickelt. ${ }^{4}$ Die persönliche Entwicklung, die Walter Eucken genommen hat, ist daher für Ökonomen äußerst ungewöhnlich: Sein Denken entwickelt sich aus der Philosophie heraus in die Ökonomik als Einzelwissenschaft hinein, wobei Eucken die Ökonomik als Denkmethode versteht und ein prononciert gesellschaftstheoretisches Erkenntnisinteresse - sowie ein prononciert gesellschaftspolitisches Gestal-

von Euckens Gesamtwerk - vgl. Pies (2000: 18-24), (2001: 3-92) und (2002). Zum Begriff einer Ordnungsethik vgl. Homann und Kirchner (1995).

3 Eucken (1950: V).

$4 \quad$ Vgl. Pies (2001: 8-31). 
tungsinteresse - verfolgt, das nicht i. e. S. wirtschaftswissenschaftlich - im Sinne einer Bereichsontologie - verkürzt ist. ${ }^{5}$

Zu den subjektiven Faktoren gehört ferner, dass Eucken seine Karriere als Einzelwissenschaftler im Umfeld der Historischen Schule der Nationalökonomie angetreten hat und aufgrund dieser wissenschaftlichen Sozialisation über ein umfängliches Reservoir nicht nur philosophiegeschichtlicher, sondern auch rechtsgeschichtlicher und insbesondere wirtschaftsgeschichtlicher Kenntnisse verfügte. Sein eigenes Denken in Ordnungen ist explizit angelehnt an das juristische Denken in politischen Verfassungen, und es ist kein Zufall, sondern Ausdruck einer bewussten Entscheidung, dass Eucken insbesondere in seiner Freiburger Zeit (1927-1950) eng mit Rechtswissenschaftlern wie beispielsweise Franz Böhm und Hans Großmann-Doerth zusammengearbeitet hat. In dieser sorgsam gepflegten Form von Interdisziplinarität kann man durchaus einen Vorläufer dessen sehen, was in den USA später als „law and economics“ entwickelt worden ist. ${ }^{6}$

$\mathrm{Zu}$ den subjektiven Faktoren gehört schließlich auch, dass Walter Eucken früh und überraschend auf einer Auslandsreise verstorben ist. Er war erst 59 Jahre alt, als er jäh aus dem Leben - und mitten aus der Arbeit - gerissen wurde. Sein ordnungspolitisches Hauptwerk war noch nicht fertiggestellt und wurde erst 1952 posthum veröffentlicht, in einer aus Notizen zusammengestellten Fassung, deren fragmentarischer Charakter nicht zu übersehen ist. Verglichen mit seinen früheren Büchern ist ausgerechnet das ordnungspolitische Hauptwerk nicht immer sorgsam durchformuliert, sondern insbesondere deshalb schwer zu lesen, weil der Argumentationsgang mitunter in mehreren Anläufen ausgeführt wird, so dass Stellen, die systematisch eng zusammengehören, im Buch teilweise weit auseinander stehen. Dadurch wird es selbst einem aufmerksamen Leser gelegentlich sehr schwer gemacht, den roten Faden dieses Buches zu erkennen und beim Lesen durchgängig im Auge zu behalten.

(2) Zu den subjektiven Rezeptionsschwierigkeiten kommen objektive hinzu. Sie resultieren vor allem daraus, dass der ökonomische Wissenschaftsbetrieb - mit durchaus guten Gründen und beeindruckenden Erfolgen - zunehmend auf eine normalwissenschaftliche Forschung ausgerichtet wird, die sich auf modelltheoretische und empirische Erkenntnisbeiträge fokussiert. Zu den unliebsamen Nebenfolgen dieser im Großen und Ganzen durchaus begrüßenswerten Entwicklung gehört, dass die Lektüre klassischer Texte mittlerweile nicht nur aus dem Studium, sondern auch aus der Ausbildung wissenschaftlicher Nachwuchskräfte fast gänzlich verschwunden ist. Man beschäftigt sich immer weniger mit den paradigmatischen Grundlagen des eigenen Fachs und konzentriert sich stattdessen auf eine immer kleinteiligere Arbeitsteilung mit entsprechenden Spezialisierungsvorteilen.

Dies hat zur Folge, dass im ökonomischen Wissenschaftsbetrieb das Verständnis genauer: die Verständnisgrundlage - für Euckens Theorieleistungen zunehmend schwindet. Denn diese Theorieleistungen sind gerade nicht auf der heute für beson-

5 Zur persönlichen Entwicklung Walter Euckens vgl. Dathe und Goldschmidt (2003) sowie Dathe (2009).

6 Vgl. hierzu die Interpretationen von Grossekettler (1997), (2000) und (2003). 
ders wichtig gehaltenen Ebene der konkreten Modellbildung angesiedelt, sondern vielmehr auf der kaum noch Aufmerksamkeit und Sorgfalt erfahrenden Ebene der Methodologie, Theoriearchitektur und Wissenschaftskonzeption. In diesem Zusammenhang ist auf drei Punkte aufmerksam zu machen:

Erstens ist Eucken als Methodologe ein wichtiger Wegbereiter der neoklassischen Modellökonomik und damit auch des Rational-Choice-Ansatzes im deutschen Sprachraum. Er hat maßgeblich mit dazu beigetragen, die bei den Anhängern der Historischen Schule weit verbreiteten und tiefsitzenden Vorbehalte gegenüber einer formalanalytisch ausgerichteten, mit mathematischen Modellen arbeitenden Ökonomik auszuräumen, die Isolation der deutschen Nationalökonomie zu überwinden und sie für die angelsächsische Analysetradition des Rationalismus zu öffnen. Der klassische Beleg hierfür ist Eucken (1934/1954).

Zweitens ist Eucken das theoriearchitektonische Meisterwerk gelungen, die ,große Antinomie zu überwinden, also den von seinen Zeitgenossen als unüberbrückbar empfundenen Widerspruch zwischen der rationalistischen Analysemethode formaler Modellbildung auf der einen Seite und dem Interesse der Historischen Schule an langfristigen gesellschaftlichen Entwicklungen aufzulösen. Eucken hält an dem historischen Erkenntnisinteresse fest, verabschiedet sich jedoch von der Idee einer historischen Längsschnittanalyse und schlägt stattdessen eine neue Vorgehensweise vor. Die besteht darin, mehrere gesellschaftliche Querschnittsanalysen hintereinanderzuschalten, nicht um die gesellschaftliche Dynamik im Zeitablauf kontinuierlich nachzuzeichnen - und hierin auf pure Deskription beschränkt zu bleiben -, sondern um sie im Wege einer komparativen Statik analytisch nachvollziehbar zu machen. Der Sache nach handelt es sich bei dieser Vorgehensweise darum, die einzelwirtschaftliche Analyse zu einer Marktformenlehre auszuarbeiten, mit deren Hilfe die Ordnungseigenschaften alternativer - historisch mannigfaltiger - Anreizarrangements untersucht werden können. Der klassische Beleg hierfür ist Eucken (1940/1959).

Drittens hat Eucken eine ordnungspolitische Konzeption entwickelt, mit deren Hilfe er das für die Theorie der Wirtschaftspolitik hochgradig relevante Grundlagenproblem adressiert, nicht der von Max Weber thematisierten Werturteilsproblematik ${ }^{7}$ anheimzufallen: Eucken entwickelt eine konzeptionell tragfähige Lösung für das Problem, wie die Ökonomik als Wissenschaft zu den extrem wertstrittigen Politikkontroversen seiner Zeit Stellung nehmen kann, ohne selbst strittige Werturteile in Anspruch nehmen zu müssen. Insofern zielt seine Ordnungspolitik nicht nur auf das Ingangsetzen und Inganghalten einer marktwirtschaftlichen Sozialstruktur, sondern auch auf eine Aufklärung der Semantik. Dies lässt sich als ein für die Einzelwissenschaft der Ökonomik eher ungewöhnlicher, aber methodologisch vorbildlicher und programmatisch wegweisender Beitrag zur Rationalisierung politischer Ideologiediskurse interpretieren. Der klassische Beleg hierfür ist Eucken (1952/1990).

Diese dritte Theorieleistung Euckens bearbeitet die Schnittstelle zwischen positiver Theorie und normativer Analyse. Hierbei gelingt ihm eine konzeptionelle Problemlösung. Die ist in der einschlägigen Sekundärliteratur bis heute weitgehend unbeachtet

Vgl. Weber (1904/1988) sowie (1918/1988). 
bzw. unverstanden geblieben. ${ }^{8}$ Deshalb konzentrieren sich die folgenden Ausführungen darauf, Euckens spezifische Problemstellung sowie seinen Problemlösungsbeitrag so zu rekonstruieren, dass die Stärken und Schwächen seines Denkansatzes deutlich werden.

\section{Walter Euckens ordnungspolitische Problemstellung}

„[D]ie Fragestellungen, die Begriffe, die Ideen und die Programme, die heute in der Welt umgehen, stammen meist aus der vergangenen Epoche, [...] aus der Zeit der sozialen Frage des 19. Jahrhunderts. [...] Die Meinungen bewegen sich noch in einer Welt, die nicht mehr real ist. [...] Der Anachronismus der herrschenden Ideen ist eine $[\ldots]$ wesentliche historische Tatsache."

(1) Walter Euckens Ordnungspolitik liegt eine Diagnose der Moderne zugrunde. Er blickt aus einer universalgeschichtlichen Perspektive auf die langfristigen Entwicklungslinien der Gesellschaft und identifiziert einen radikalen Bruch. Hierzu schreibt er in der Mitte des 20. Jahrhunderts:

„Industrialisierung und moderne Technik haben einen einzigartigen Umsturz in der Geschichte bewirkt. Die wirtschaftlich-technische Umwelt jedes einzelnen Menschen hat sich völlig geändert; die Umwelten Goethes und Platons waren einander ähnlicher als die Umwelten Goethes und eines heute lebenden Menschen. “10

Für Eucken haben die Industrialisierung und die moderne Technik eine epochale Wirkung nicht nur im Hinblick auf den wirtschaftlichen Reichtum, den sie ermöglichen, sondern vor allem im Hinblick auf die gesamtgesellschaftlichen Konsequenzen, die mit ihnen verknüpft sind. Diese reichen von der individuellen Freiheit des einzelnen Menschen - insbesondere der Möglichkeit, ein selbstbestimmtes Leben zu führen bis hin zur Möglichkeit einer rechtsstaatlichen und demokratischen Verfassung.

Aus Euckens Sicht ist das Potential der Moderne in dreierlei Hinsicht nicht genutzt worden. Im Rückblick auf die erste Hälfte des 20. Jahrhunderts spricht er von ,unserem Zeitalter der Misserfolge“ (so der programmatische Titel bei Eucken 1951) und meint damit, dass die moderne Gesellschaft sowohl wirtschaftlich als auch moralisch und politisch unter ihren Möglichkeiten geblieben ist. Gerade in den Jahren unmittelbar nach dem Zweiten Weltkrieg ist für ihn unabweisbar, dass von Grund auf neue Lösungen gefunden werden müssen, die erstens die wirtschaftliche Knappheit erfolgreich bewältigen, zweitens dem Individuum eine moralische Selbstbestimmung erlauben und drittens eine dauerhaft stabile rechtsstaatliche Demokratie ermöglichen. Eucken hat aus seinem universalhistorischen Blickwinkel explizit diese drei Dimensionen vor Augen, wenn er das Problem seiner Ordnungspolitik „als ein Zentralproblem unseres Zeitalters“ (Eucken 1948a: 56) mit folgender Formulierung kennzeichnet:

\footnotetext{
8 Vgl. Pies (2001: 69-92, mit zahlreichen Literaturnachweisen).

$9 \quad$ Eucken (1948a: 120f., H. i. O.).

$10 \quad$ Eucken (1948a: 56).
} 
„Wie kann dieser modernen industrialisierten Wirtschaft und Gesellschaft eine menschenwürdige und funktionsfähige Ordnung gegeben werden?" "11

Das wichtigste und drängendste Problem der Wirtschaftspolitik bestimmt Eucken mithin als Problem einer Wirtschaftsordnungspolitik, die zugleich Gesellschaftsordnungspolitik ist. Beides gehört für ihn eng zusammen, weil er davon ausgeht, dass es eine "Interdependen₹ der Ordnungen"12 gibt, so dass man gleichzeitig Wirtschaft und Gesellschaft im Blick haben muss, um die jeweiligen Rückwirkungen zwischen den Ordnungen berücksichtigen zu können.

(2) Eucken formuliert aber nicht nur die ordnungspolitische Problemstellung als Desiderat einer Wirtschafts- sowie Gesellschaftsverfassung, sondern er identifiziert zugleich ein Hindernis, das der einfachen Lösung dieses Problems im Wege steht. Es gehört konstitutiv zu seiner Diagnose der Moderne, dass das Ordnungsproblem nicht nur darin besteht, eine (Re-)Formierung institutioneller Anreizarrangements herbeizuführen, sondern auch darin, Denkblockaden zu überwinden, die die erforderlichen Weichenstellungen behindern. Hierzu liest man:

„Während im Zeitalter der Industrialisierung das wirtschaftsordnungspolitische Problem fortwährend an Gewicht gewinnt, nimmt die Fähigkeit, das Problem zu sehen und zu behandeln, mehr und mehr ab. Das Erfordernis der Sache und die Fähigkeit, der Sache gerecht zu werden, bewegen sich in entgegengesetzter Richtung." 13

Eucken identifiziert hier ein Problem intellektueller Orientierung: Seiner Auffassung zufolge wird der Blick auf die ordnungspolitische Fragestellung von Ideologien zugestellt, die seit geraumer Zeit tendenziell verhindert haben, dass die richtigen Antworten gefunden werden können. Mitte des 20. Jahrhunderts lautet seine Diagnose:

„[D]ie wirtschaftspolitischen Doktrinen, welche die meisten Menschen heute beherrschen und die auch maßgebend für die Wirtschaftspolitik sind, wurden bereits vor langer Zeit entwickelt: Es sind merkantilistische Ideen des 18. Jahrhunderts, liberale des 18. und 19. Jahrhunderts und sozialistische, die im Wesentlichen während der ersten Hälfte des 19. Jahrhunderts entstanden sind. [...] Die Ideen von damals, die noch heute herrschen, sind alt und veraltet. So besteht eine Diskrepanz zwischen Realität und wirtschaftspolitischen Ideologien." ${ }^{14}$

(3) Aus diesem Befund leitet Eucken eine spezifische Aufgabenstellung für die Wissenschaft ab. Sie soll dazu beitragen, den ideologischen Schleier zu durchstoßen und den Blick auf das ordnungspolitische Problem freizulegen. Ihm geht es darum, den im einleitenden Motto zu Beginn dieses Abschnitts zitierten „Anachronismus der Ideen“ wissenschaftlich zu überwinden und Aufklärungsleistungen zu generieren, die den drei Dimensionen sind, von denen aus man auf das Ordnungsproblem stößt.

12 Eucken (1948a: 72, H. i. O.).

13 Eucken (1948a: 78).

$14 \quad$ Eucken (1951:2). 
Bürgern eine intellektuelle Orientierungshilfe geben und sie zum „Denken und Handeln in Ordnungen" (Eucken 1948a: 90) befähigen.

Im Hinblick auf die intendierte Orientierungsleistung der Wissenschaft sind zwei Punkte von besonderer Bedeutung. Wenn man sie nicht klar vor Augen hat, fällt es schwer, Euckens Ansatz richtig zu verstehen.

Erstens soll die Ökonomik Eucken zufolge ihren Aufklärungsbeitrag primär als Erfahrungswissenschaft leisten: Seine Diagnose der Moderne sieht die Situation der Zeit bestimmt durch eine Diskrepanz zwischen Realität und Ideologie, und diese Diskrepanz soll von wissenschaftlicher Seite vor allem dadurch abgebaut werden, dass die mittlerweile verfügbaren Erkenntnisse über die Folgen ideologischer Optionen der Öffentlichkeit zugänglich gemacht werden. Hierzu liest man:

„In einer solchen Situation ist es notwendig, die Ideologien von ehedem beiseite zu schieben und die Erfahrung vollständig zu Geltung zu bringen. Gerade das letzte halbe Jahrhundert hat mit seinem raschen Wechsel der Wirtschaftspolitik, mit dem Interventionismus, der Vollbeschäftigungspolitik, den zentralverwaltungswirtschaftlichen Versuchen in Russland, Deutschland und anderen Ländern viel gelehrt. Seit 1914 experimentierte man mit neuen Währungsformen, mit Marktregelungen, Selbstverwaltung der Wirtschaft, Korporationen, zentraler Planung usw. - ohne zu wissen, was dabei herauskommen werde. Heute ist das anders. Die Erfahrungen mit solchen Experimenten liegen vor. [...] Diese Erfahrungen auszuschöpfen und für die Zukunft nutzbar zu machen, ist eine wesentliche Aufgabe der Wissenschaft.“"15

Zweitens kommt Euckens Vorstellung einer ökonomischen Aufklärung ein Umstand zu Hilfe, auf den er erstmals schon 1932 hingewiesen hatte. Im Hinblick auf die wirtschaftspolitischen Doktrinen des Interventionismus und der zentralen Planwirtschaft konstatiert er:

\begin{abstract}
„Mit diesen Ideologien hat es nun aber eine eigentümliche Bewandtnis: Sie bejahen und befürworten nämlich Bewegungen, die genau das Gegenteil von dem erreichen, was die Ideologen von ihnen erhoffen. [...] Die Interventionisten wollen entweder Stärkung des Staates oder Ordnung der Volkswirtschaft oder beides; deshalb bekämpfen sie den liberalen Staat und treten für Interventionismus und Wirtschaftsstaat ein, die [...] genau zum Gegenteil dessen geführt haben und führen, was die Ideologen erstreben: nämlich zur Schwächung des Staates und zur Desorganisation der Volkswirtschaft."16
\end{abstract}

Eucken setzt also ganz offenkundig schon seit 1932 darauf, dass die Ökonomik durch eine Untersuchung der nicht-intendierten Folgen intentionalen Handelns zur Aufklärung von Ideologien beitragen kann, indem sie die geschichtlich verfügbaren Erfahrungen analysiert und theoretisch durchdringt. In diesem Sinne ist seine frühe Diagnose des doppelten Defizits zeitgenössischer Ideologien ausgesprochen programmatisch:

\footnotetext{
$15 \quad$ Eucken (1951: 2f., H. i. O.).

16 Eucken (1932: 319, H. i. O.).
} 
„Es fehlt der Sinn für geschichtliche Tatsachen, es fehlt das strenge ökonomische Denken. “"17

(4) Die bisherige Argumentation hat deutlich werden lassen, dass das ordnungspolitische Verfassungsproblem einer konstitutionellen (Re-)Formierung von Wirtschaft und Gesellschaft für Eucken nicht nur eine sozialstrukturelle, sondern auch eine semantische Dimension aufweist, weil aus seiner Sicht die Schlagworte, Begriffe und Denkkategorien der zeitgenössischen Auseinandersetzung ideologisch verzerrt sind und deshalb einer wissenschaftlichen Aufklärung bedürfen. Der folgende Abschnitt rekonstruiert, wie Eucken sich das konkret vorgestellt hat und welchen Aufklärungsbeitrag er als Ökonom zu leisten bestrebt war.

\section{Walter Euckens ordnungspolitische Problembearbeitung}

„Sobald die Lenkungsmechanik der modernen Wirtschaft nicht funktioniert, verschärft sich die soziale Frage." ${ }^{18}$

Walter Eucken hat in den letzten 18 Jahren seines Lebens - von 1932 bis 1950 - eine ordnungspolitische Konzeption entwickelt, die die Argumentationsstruktur einer Gesellschaftsvertragstheorie aufweist - also lange bevor James Buchanan mit seiner Demokratietheorie und John Rawls mit seiner Gerechtigkeitstheorie die in der praktischen Philosophie lange Zeit für tot erklärte Tradition eines gesellschaftsvertragstheoretischen Denkens wieder belebt bzw. wiederbelebt haben. ${ }^{19}$ Abbildung 1 hilft dabei, sich Euckens Argumentation vor Augen zu führen.

Für den fiktiven Zeitpunkt $t_{1}$ wird eine Verfassungsgebung angesetzt. Eucken (1952/1990: 305) verwendet hierfür den Begriff einer ,wirtschaftsordnungspolitischen Gesamtentscheidung“. Seine Theorie soll sowohl ex ante als auch ex post einen prononciert wissenschaftlichen Beitrag hierzu leisten. Ex ante geht es darum, die Alternativen und die Kriterien für die Gesamtentscheidung zu spezifizieren, während es ex post - nach einer bereits erfolgten Gesamtentscheidung - darum geht, die im Verfassungsentscheid gebilligte Ordnung konsequent zu implementieren.

$17 \quad$ Eucken (1932: 321).

$18 \quad$ Eucken (1952/1990: 187).

19 Vgl. Buchanan und Tullock (1962, 1990) sowie Rawls (1971, 1979). Für einen Überblick hierzu vgl. Homann (1988). - Interessanterweise benötigt Eucken für seine Argumentation weder einen Schleier der Unsicherheit wie Buchanan noch einen Schleier des Nichtwissens wie Rawls. 


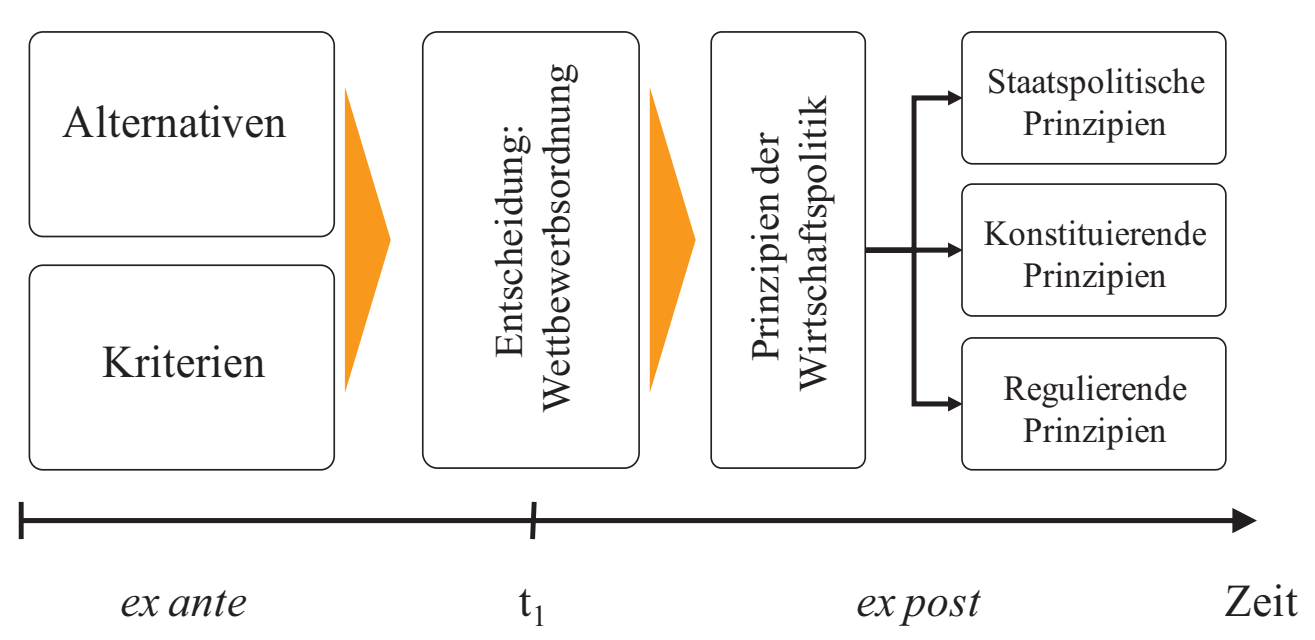

Abbildung 1: Euckens Argumentationsfigur eines Gesellschaftsvertrags20

(1) Im Hinblick auf die zur Entscheidung stehenden Alternativen geht Eucken von dem Befund aus, dass sich im zeitgenössischen Diskurs zwei radikale Positionen gegenüberstehen: auf der einen Seite ein Laissez-faire-Liberalismus, der ganz auf die Selbststeuerungskräfte des Marktes setzt und eine staatsfreie Wirtschaft propagiert; sowie auf der anderen Seite ein Zentralplanungs-Sozialismus, der den Markt ablehnt und stattdessen eine Staatswirtschaft propagiert.

Eucken selbst hält diese Frontstellung für verkehrt. Er macht geltend, dass die zeitgenössische Auseinandersetzung einen blinden Fleck aufweise, weil übersehen werde, dass es eine dritte Option gebe. Zur Überwindung des eindimensionalen Streits zwischen radikalen Extremen setzt Eucken auf eine zweidimensionale Betrachtung. Er unterscheidet systematisch zwischen Form und Prozess des Wirtschaftens - in der Sprache des Sports: zwischen Spielregeln und Spielzügen. Abbildung 2 hilft, sich vor Augen zu führen, wie Eucken mit dieser Zwei-Ebenen-Unterscheidung das Spektrum wirtschafts- und gesellschaftspolitischer Optionen erweitert und die Aufmerksamkeit auf eine dritte Option lenkt, die bislang gar nicht ins Blickfeld der zeitgenössischen Auseinandersetzung geraten war. 


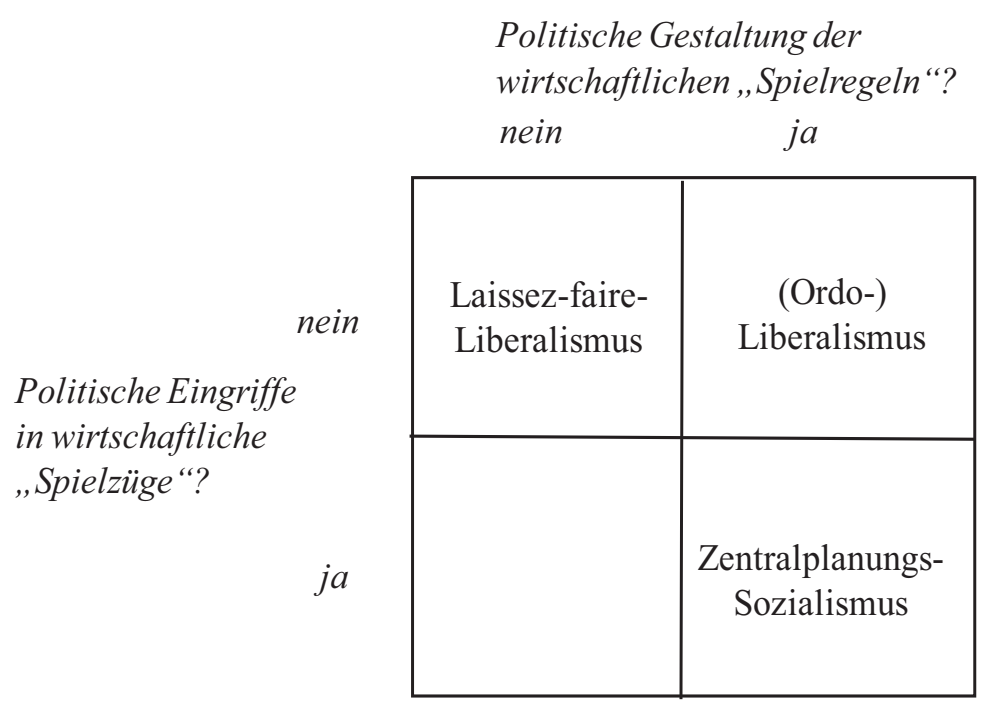

Abbildung 2: Tertium datur: Euckens dritte Option

Euckens konstruktiv(istisch)e Argumentation zugunsten des Ordo-Liberalismus kommt besonders deutlich in folgender Passage zum Ausdruck:

„Soll der Staat wenig oder viel tun? Wenig, so antworten die Anhänger des Laissezfaire. Viel, - sagen die Anhänger der Wirtschaftspolitik zentraler Planung. Einen Mittelweg suchen die Freunde von Kompromisslösungen. [...] Aber das Problem sollte anders gestellt werden, um lösbar zu sein. Ob wenig oder mehr Staatstätigkeit, diese Frage geht am Wesentlichen vorbei. Es handelt sich nicht um ein quantitatives, sondern um ein qualitatives Problem. [...] Welcher Art also sollte die Staatstätigkeit sein? Die Antwort lautet: Der Staat hat die Formen, in denen gewirtschaftet wird, zu beeinflussen, aber er hat nicht den Wirtschaftsprozess selbst zu führen. [...] Staatliche Planung der Formen - ja; staatliche Planung und Lenkung des Wirtschaftsprozesses - nein. Den Unterschied von Form und Prozess erkennen und danach handeln, das ist wesentlich. ${ }^{\text {" }} 21$

Um diese Passage richtig verstehen und einordnen zu können, muss zunächst geklärt werden, an welchen Kriterien sich Eucken orientiert, wenn er die Empfehlung abgibt, die wirtschaftsordnungspolitische Gesamtentscheidung jenseits des Gegensatzes von Laissez-faire und zentraler Planung zugunsten der ordo-liberalen Option einer Wettbewerbsordnung zu treffen, in der die Rahmenordnung politisch festgelegt wird und die einzelwirtschaftlichen Entscheidungen eigenverantwortlich unter Wettbewerbsdruck stattfinden.

Prinzipiell kommen für Eucken drei Werte in Frage, die man bei der Verfassungsgebung als Entscheidungskriterium zugrunde legen könnte: Freiheit, soziale Gerech- 
tigkeit und soziale Sicherheit. Nun ist sich Eucken des - im Hinblick auf Max Webers Werturteilsfreiheitspostulats: misslichen - Umstands sehr bewusst, dass die Laissezfaire-Liberalen für ihre Sache den Wert der Freiheit ins Feld führen, während die Zentralplanungs-Sozialisten für ihre Sache die Werte der sozialen Sicherheit und sozialen Gerechtigkeit reklamieren. Zunächst sieht es also so aus, als müsse man sich nicht nur zwischen den beiden Positionen der staatsfreien Wirtschaft und der Staatswirtschaft entscheiden, sondern auch zwischen den (vermeintlich) zugehörigen Werten. Hiergegen setzt Eucken eine Argumentation, die drei Varianten der sozialen Frage unterscheidet und auf die These hinausläuft, dass im Hinblick auf die relevanten Alternativen der wirtschaftsordnungspolitischen Gesamtentscheidung eine wertstrittige Stellungnahme erübrigt und dennoch eine eindeutige Empfehlung ausgesprochen werden kann.

Die erste Variante der sozialen Frage - das Problem sozialer Gerechtigkeit - datiert Eucken auf die Zeit vom Beginn der Industrialisierung bis 1914. Hier steht die Ungleichheit im Vordergrund, vor allem in Form eines niedrigen Lebensstandards der Arbeiter. Die staatliche Reaktion hierauf ist eine umfassende Sozialpolitik. Deren Spektrum reicht von der Arbeitsschutzgesetzgebung bis hin zur Einrichtung kollektiver Systeme zur Abdeckung von Krankheits- und Unfallrisiken. ${ }^{22}$ Die zweite Variante der sozialen Frage - das Problem sozialer Sicherheit - datiert Eucken von 1914 bis zur Mitte der 1930er Jahre. Hier steht die Massenarbeitslosigkeit im Vordergrund. ${ }^{23}$ Die staatliche Reaktion hierauf ist eine neue Form der Sozialpolitik, die auf Vollbeschäftigung abzielt, zu diesem Zweck den Markt weitgehend außer Kraft setzt und stattdessen zentralplanerische Elemente in die Wirtschaftsordnung einführt. Die Staatswirtschaft des totalen Wirtschaftsstaats zeitigt totalitäre Folgen und leitet damit die aus Euckens Sicht dritte Variante der sozialen Frage ein. Hier steht die Gefährdung individueller Freiheit im Vordergrund.

Die politische Reaktion auf den Wechsel von der ersten zur zweiten sozialen Frage hat aus Euckens Sicht zu einer Verschlimmbesserung geführt, die ihrerseits die dritte soziale Frage ausgelöst hat: Im Totalitarismus zentraler Planung gibt es keine individuelle

22 Hierzu liest man bei Eucken (1951: 32) folgende Einschätzung: „Erfolge hat diese Sozialpolitik zweifellos gehabt. Aber die außerordentliche Steigerung des Lebensstandards der Arbeiter, die sich im Verlauf des 19. Jahrhunderts und zu Beginn des 20. Jahrhunderts vollzog, ist nicht in erster Linie auf die Sozialpolitik zurückzuführen. Denn die Erhöhung des Lebensstandards war hauptsächlich das Ergebnis der wirtschaftlich-technischen Entwicklung selbst. Je besser die Ausrüstung der Arbeiter mit Maschinen wurde, je größer also das Produkt des Arbeiters war, desto höher konnte der Lohn steigen."

23 Hierzu liest man bei Eucken (1951: 32f., H. i. O.): „Im Zeitalter der Experimente, also nach dem ersten Weltkrieg, erhielt die soziale Frage einen neuen Charakter. Früher war es vor allem die Ungerechtigkeit der Verteilung, welche den sozialpolitischen Eingriff des Gesetzgebers hervorrief. Jetzt rückte ein neues Problem ganz in den Vordergrund: Die Unsicherbeit in der besonderen Form der lang andauernden Arbeitslosigkeit von Massen. Wir dürfen nicht vergessen, dass es diese Form der Arbeitslosigkeit in den Jahrzehnten vor 1914 nicht gegeben hat. Nun aber wurde sie zu einer Tatsache, welche die Sozial- und Wirtschaftspolitik [...] zu beherrschen begann." Vgl. auch die ausführliche abschließende Kennzeichnung bei Eucken (1951: 40). 
Freiheit, und zudem gibt es dort weder soziale Sicherheit noch soziale Gerechtigkeit. ${ }^{24}$ Vor diesem Hintergrund dekonstruiert Eucken den vermeintlichen Wertekonflikt.25 Wer sich für die Wettbewerbsordnung entscheidet, muss dies Eucken zufolge nicht auf die wertstrittige Entscheidung gründen, der Freiheit den Vorzug zu geben vor Gerechtigkeit und Sicherheit, ganz im Gegenteil. Eucken argumentiert, dass Freiheit eine Vorbedingung ist, ohne die es soziale Sicherheit und soziale Gerechtigkeit gar nicht geben kann:
„Damit [...] stoßen wir bei der Analyse der deutschen Erfahrungen auf den Kernpunkt der sozialen Frage: Die Freiheit. Die Unfreiheit ermöglichte den Druck auf die Daseinsbedingungen. Die Herrschaft privater oder öffentlicher Machtkörper gefährdete die Durchsetzung der Gerechtigkeit; und mangelnde Sicherheit entsprang aus mangelnder Freiheit. Ohne Freiheit der Person ist die soziale Frage nicht zu lösen. “26

Nimmt man diese Überlegungen zusammen, dann lautet Euckens ordnungspolitische Empfehlung, dass man sich sowohl aus Gründen sozialer Sicherheit und sozialer Gerechtigkeit als auch insbesondere aus Gründen individueller Freiheit gegen eine zentrale Planung und stattdessen für eine Wettbewerbsordnung entscheiden sollte. Die ordnungspolitische Option, sich für eine Verfassung zu entscheiden, in der der Staat nicht einfach die Wirtschaft übernimmt, sondern sich differenziert verhält, indem nur die institutionellen Rahmenbedingungen des Marktes - die Spielregeln - einer politischen Gestaltung unterworfen werden, während der eigentliche Wirtschaftsprozess die Wahl der Spielzüge - in je individueller Verantwortung abläuft, erscheint Eucken in jeder der drei Hinsichten als eindeutig vorzugswürdig, so dass bei diesem Entscheidungsproblem ein Wertekonflikt gar nicht besteht - mit folgender Begründung: In ihrer modernsten Form besteht die soziale Frage primär in einer Gefährdung individueller Freiheit durch die staatliche Macht eines totalitären Staates, der sich mit einer zentralen Planung der Wirtschaft übernimmt. Für Eucken bietet die Wettbewerbsordnung demgegenüber nun einen doppelten Vorteil: Erstens löst sie das Problem staatlicher Machtkonzentration, weil der Staat wirtschaftlich so entlastet wird, dass er als Rechtsstaat funktionieren kann und nicht länger darauf angewiesen ist, seine Anordnungen diktatorisch umzusetzen. Die Umstellung von Subordination auf Koordination kommt der individuellen Freiheit unmittelbar zugute. Zugleich aber löst die Wett-

24 In dieser Hinsicht verweist Eucken (1951: 37) auf historische Erfahrungen: „,Wir können Punkt für Punkt feststellen, wie sich die Lage der Arbeiter bei einer Politik zentraler Lenkung des Wirtschaftsprozesses in Deutschland geändert hat. Drei Momente sind besonders wichtig: Unzureichende Versorgung der Arbeiter, Ungerechtigkeit der Verteilung und schließlich Unsicherheit."

25 So liest man bei Eucken (1951: 39): „Es ist üblich, die »Sicherheit« der »Freiheit« gegenüberzustellen. Sicherheit erfordere den Verzicht auf Freiheit. Freiheit sei dem modernen Menschen ziemlich gleichgültig. Trachte er doch vor allem nach Sicherheit. [...] Aber die deutschen Erfahrungen haben gezeigt, dass dieser Gegensatz von Sicherheit und Freiheit garnicht besteht."

26 Eucken (1951: 39f.). Präzisierend liest man bei Eucken (1948b: 129): „Ohne Freizügigkeit, freie Wahl des Arbeitsplatzes, freien Arbeitsvertrag und Auflösung oder Schwächung einseitiger wirtschaftlich-sozialer Machtpositionen kann es keine Lösung der sozialen Frage geben.“ 
bewerbsordnung, zweitens, auch das Problem privater Machtkonzentration, weil der Wettbewerb als Entmachtungsinstrument wirtschaftlicher Akteure eingesetzt wird, mit ebenfalls freiheitsfördernden Konsequenzen. Es ist diese Doppelwirkung der Wettbewerbsordnung, der gleichzeitige Abbau staatlicher und privater Machtkonzentration, den Eucken vor Augen hat, wenn er den Anspruch erhebt, dass (nur) sie in der Lage sei, die soziale Frage im Geist der Freiheit zu beantworten. ${ }^{27}$

(2) Mit diesem Nachweis der generellen Vorzugswürdigkeit einer Wettbewerbsordnung wechselt Euckens ordnungspolitische Argumentation vom Ex-ante-Stadium zum Ex-post-Stadium seiner Gesellschaftsvertragstheorie (Abb. 1).

„Bei Anwendung der wesentlichen Kriterien und in Würdigung der gegebenen
geschichtlichen Gesamtsituation bleibt allein die Wettbewerbsordnung, welche das
ordnungspolitische Problem zu lösen vermag. Wenn aber für sie die Gesamtent-
scheidung fällt, so fragt es sich: Was hat zu geschehen, um sie zu verwirklichen?"28

Auch hier macht Eucken geltend, dass es primär historische Erfahrungen sind, die er theoretisch verarbeitet. Zu diesem Zweck stellt er Grundsätze auf, an denen man sich zweckmäßigerweise orientieren sollte, wenn man ernsthaft anstrebt, eine funktionsfähige Wettbewerbsordnung in Gang zu setzen und nachhaltig in Gang zu halten. Eucken reklamiert hier für sich eine Analogie zur Staatslehre, die das Prinzip der Gewaltenteilung aufgestellt hat, um einen Rechtsstaat zu konstitu(tionalis)ieren:

„Parallel argumentieren wir: Wenn die Wettbewerbsordnung realisiert werden soll, so sind die nunmehr zu entwickelnden Prinzipien anzuwenden. Ebenso wie das Prinzip von der Teilung der Gewalten aus der geschichtlichen Erfahrung über den Missbrauch der Exekutive entstanden ist - so sind auch die Prinzipien, deren Verwirklichung die Wettbewerbsordnung konstituiert, aus der wirtschaftspolitischen und wirtschaftlichen Erfahrung gewonnen. “29

Insgesamt sind es drei Arten von Grundsätzen, die Eucken aufstellt: Erstens handelt es sich um zwei staatspolitische Grundsätze, die der Wirtschaftspolitik im engeren Sinne vorangestellt werden. Zweitens handelt es sich um sieben konstituierende Grundsätze, die dem Aufbau einer Wettbewerbsordnung als Richtschnur dienen sollen. Und drittens schließlich handelt es sich um regulierende Grundsätze, die der Aufrechterhaltung einer bereits eingerichteten Wettbewerbsordnung Orientierung geben sollen. ${ }^{30}$

27 Das Argument von Eucken (1952/1990: 332-338) ist sogar noch differenzierter: Für ihn gehört es zur Interdependenz der Ordnungen, dass vom Wettbewerb nicht nur wirtschaftliche Vorteile ausgehen, sondern auch der politische Vorteil, wirtschaftliche Machtkonzentrationen zu vermeiden, die ansonsten auf die Politik übergreifen können und dann drohen, Rechtsstaat und Demokratie außer Kraft zu setzen. Deshalb ist seine Wirtschaftsordnungspolitik schon vom Ansatz her eine umfassende Gesellschaftsordnungspolitik.

$28 \quad$ Eucken (1949: 28).

$29 \quad$ Eucken (1949: 31).

30 Vgl. - in dieser Reihenfolge - Eucken (1952/1990: 334-337, 254-291 und 291-304). Die staatspolitischen Grundsätze werden in der Sekundärliteratur oft übersehen, weil sie in Euckens posthum zusammengestelltem Buch den konstituierenden und regulierenden Prinzipien nicht vorgeschaltet, sondern nachgeschaltet sind, und dies auch noch mit deutlichem Platzabstand. Hier zeigt sich besonders deutlich der fragmentarische Charakter seines wirtschaftspolitischen 
Für die hier interessierende Fragestellung ist es nicht nötig, die wirtschaftspolitischen Grundsätze im Detail zu erörtern. Aber es sei doch wenigstens darauf hingewiesen, dass Eucken den Anspruch erhebt, mit diesen Grundsätzen eine in sich geschlossene Konzeption zur intellektuellen Orientierung der Wirtschafts- und Gesellschaftspolitik vorgelegt zu haben:

„Alle Prinzipien - die konstituierenden und die regulierenden - gehören zusammen. [...] Jedes einzelne Prinzip erhält nur im Rahmen des allgemeinen Bauplanes der Wettbewerbsordnung seinen Sinn. [...] Die einzelnen Prinzipien ergänzen einander, sind komplementär.“" ${ }^{31}$ Und an anderer Stelle heißt es: „Die beiden Grundsätze der Staatspolitik [...] harmonieren [...] vollständig mit den Prinzipien, die für den Aufbau einer zureichenden Wirtschaftsordnung gelten.“32

Wichtig ist auch, sich den Status zu vergegenwärtigen, den Eucken seinen Grundsätzen zuweist: Einerseits bedient sich Eucken der philosophischen Gedankenfigur eines Gesellschaftsvertrags, um seine wissenschaftliche Stellungnahme zum wohl drängendsten Politikproblem seiner Zeit mit einer diskursiv leistungsfähigen Struktur zu versehen. Der Grundgedanke seiner Ordnungspolitik lässt sich auf folgenden Satz zuspitzen: Wenn es auf eine für alle Bürger einsehbare Weise zweckmäßig ist, sich gegen eine Zentralplanung und für eine Wettbewerbsordnung zu entscheiden, dann ist es auch auf eine für alle Bürger einsehbare Weise konsequent, den diesem Zweck dienlichen Grundsätzen der Wirtschaftspolitik zuzustimmen.

Andererseits setzt sich Eucken ganz deutlich von der philosophischen Tradition ab, wenn es darum geht, die eigene Argumentationsgrundlage auszuweisen. Er macht für sich geltend, die Kenntnisse der ökonomischen Erfahrungswissenschaft in reine Zweckmäßigkeitsargumente zu übersetzen.

„Die konstituierenden Prinzipien sind Prinzipien der Wirtschaftsverfassung. [...] [Sie] dienen also einer wirtschaftspolitischen Gesamtentscheidung und sind Mittel, um die Gesamtentscheidung in concreto durchzusetzen. “33 Gleich im Anschluss heißt es weiter: „Es handelt sich also nicht um rechtsdogmatische und nicht um naturrechtliche Prinzipien. Einzelne Prinzipien - wie das Prinzip der Vertragsfreiheit oder der Haftung oder des Privateigentums - sind von Philosophen und Rechtsdenkern auch als reine Rechtsprinzipien entwickelt worden; etwa als Prinzipien des Naturrechts. Aber aus dem Naturrecht oder aus übergeordneten rechtsdogmatischen Sätzen sind sie in dem Ordnungszusammenhang, von dem hier die Rede ist, nicht abgeleitet. Zum Beispiel wird die Forderung, Privateigentum herzustellen, vielfach aus der Natur des Menschen deduziert und als ein Gebot des Naturrechts begründet. - Hier geschieht dies nicht; Privateigentum erwies sich als notwendig, um - zusammen mit den übrigen Prinzipien - eine Wettbewerbs-

Hauptwerks. Bei Eucken (1949) finden sich übrigens nur die konstituierenden und regulierenden Prinzipien. Dies deutet darauf hin, dass er noch kurz vor seinem Tod mit der vollen Ausarbeitung seiner Grundsätze beschäftigt gewesen sein muss.

$31 \quad$ Eucken (1952/1990: 304).

32 Eucken (1952/1990: 327, im Original teilweise hervorgehoben).

33 Eucken (1951: 62, H. i. O.). 
ordnung zu konstituieren. [...] Auf dem positiven Zweck liegt bei allen diesen Prinzipien der Nachdruck. “34

Damit stellt Eucken unmissverständlich klar, dass er sein Plädoyer für die Wettbewerbsordnung einzig und allein auf das Wollen der Bürger gründet. Ihnen gegenüber gibt es keine externe Autorität, von der aus Verbindlichkeit postuliert werden könnte. Innerhalb des Diskurses, den Eucken führt, gibt es nur die interne Autorität der Bürger, deren aufgeklärtes Ordnungswollen zur Geltung gebracht werden soll, nachdem genau dies ein halbes Jahrhundert lang immer wieder gescheitert ist - im Zeitalter der Experimente, das für Eucken ein Zeitalter der Misserfolge und der totalitären Freiheitsbedrohung war. Deshalb entwickelt Eucken seine Theorie als einen wissenschaftlichen Aufklärungsbeitrag. Denn aus seiner Sicht ist es „notwendig, dass die Träger der Ordnung auch den Sinn der Ordnung verstehen“"35.

\section{Ordnungsethik und Ordnungspolitik bei Walter Eucken}

„Die Gesamtordnung sollte so sein, dass sie den Menschen das Leben nach ethischen Prinzipien ermöglicht. "36

Die bisherige Argumentation hat deutlich werden lassen, wie Eucken das Problem intellektueller Fehlorientierung mit seiner ordnungspolitischen Konzeption zu bearbeiten versucht hat. Auf dieser Grundlage lässt sich nun der Frage nachgehen, in welchem Verhältnis Ordnungsethik und Ordnungspolitik bei Walter Eucken zueinander stehen. Hierbei soll deutlich werden, dass triftige Gründe für die im Titel dieses Beitrags zum Ausdruck gebrachte These sprechen, dass Walter Eucken nicht nur als Klassiker der Ordnungspolitik, sondern ganz dezidiert auch als Klassiker der Ordnungsethik zu würdigen ist.

Für diese These sind nun zwei Belege ins Feld zu führen. Der erste Beleg rekurriert darauf, dass Eucken einen auch heute noch unvermindert aktuellen Grundlagenbeitrag zur Ordnungsethik - und sogar noch allgemeiner: zur Moralphilosophie generell geleistet hat. Ordonomisch rekonstruiert, lässt sich seine Argumentation als eine orthogonale Positionierung 37 interpretieren, die die eindimensionale Entgegensetzung von Egoismus und Altruismus bzw. von Eigennutz und Gemeinwohl überwindet und damit einer intellektuellen Begriffsverwirrung entgegentritt, die auch heute noch viel Unheil stiftet. Der zweite Beleg rekurriert darauf, dass Eucken die Beziehung zwischen Ordnungsethik und Ordnungspolitik explizit behandelt und hierbei eine sehr differenzierte Verhältnisbestimmung vornimmt, die letztlich darauf hinausläuft, die Wirtschafts- und Gesellschaftsordnung an einem ethischen Ordnungswollen auszurichten.

\footnotetext{
$34 \quad$ Eucken (1951: 62f.).

35 Eucken (1952/1990: 370).

36 Eucken (1952/1990: 199, im Original hervorgehoben).

$37 \quad$ Als ,orthogonale Positionierung“ wird ein Wechsel der Denkrichtung bezeichnet, der aus einer eindimensionalen Konfliktwahrnehmung aussteigt, indem eine zweite Dimension hinzugedacht wird, die es ermöglicht, auch gemeinsame Interessen zu identifizieren. Vgl. hierzu Pies (2000: 34f. et passim).
} 
(1) In der Sekundärliteratur ist es weitgehend unbeachtet geblieben, dass Walter Eucken sich explizit und ausführlich zu Fragen der Ethik geäußert hat. ${ }^{38}$ Der klassische Beleg hierfür ist Eucken (1952/1990: 350-371, Kapitel XX). Bei der nun folgenden Rekonstruktion seines ordnungsethischen Theoriebeitrags steht im Vordergrund, wie Eucken seine Argumentation schrittweise entwickelt. Insgesamt lassen sich drei Schritte nachzeichnen.

Erster Schritt: Eucken referiert zwei Positionen, die sich diametral widersprechen. Er identifiziert folgende Frontstellung: Die erste Position vertrete die Auffassung, dass das Streben nach Eigennutz dem Gemeinwohl abträglich sei, während die zweite Position die Gegenauffassung vertrete, dass das Streben nach Eigennutz dem Gemeinwohl zuträglich sei. Die zweite Auffassung behauptet eine Harmonie der Interessen, während die erste Auffassung eine Disharmonie behauptet. Für beide Behauptungen lassen sich einzelne Beispiele anführen. Mit diesem Befund sieht Eucken die Debatte in einer extrem wichtigen Frage blockiert:

„Die Erfahrung scheint also beiden Seiten recht zu geben, und das Problem scheint prinzipiell unlösbar zu sein. - Aber eine Klärung ist notwendig, ob die eine oder andere Seite im Recht ist, warum und wie. Nur dann kann Wirtschaftspolitik ernsthaft getrieben werden. ${ }^{\text {“39 }}$

Zweiter Schritt: Eucken führt mehrere Differenzierungen ein, die einer Überwindung des diametralen Gegensatzes - und der durch ihn ausgelösten Diskurs- und Erkenntnisblockade - vorarbeiten.

Zunächst einmal unterscheidet er im Hinblick auf moralische Motive zwischen Egoismus und Altruismus, und zwar ganz in klassischer Manier: Egoismus wird begrifflich gefasst als eigeninteressiertes Handeln ohne jede Rücksicht auf die Interessen anderer, während Altruismus dadurch gekennzeichnet wird, dass hier eine Rücksichtnahme auf die Interessen anderer erfolgt. Der Egoist handelt auf Kosten seiner Mitmenschen, der Altruist handelt (auch) zu ihren Gunsten. ${ }^{40}$

Sodann führt Eucken eine Ebenen-Unterscheidung ein zwischen moralischen Motiven auf der einen Seite und dem wirtschaftlichen Prinzip rationalen Handelns auf der anderen Seite. Hierzu liest man:

„Mit Egoismus oder Altruismus hat das wirtschaftliche Prinzip gar nichts zu tun.

[...] Die dauernde Vermischung von »Egoismus« und »wirtschaftlichem Prinzip« ist der Krebsschaden der ganzen Diskussion über diesen wichtigen Problemkomplex. [...] Egoismus oder Altruismus bestimmen die Zwecke, auf welche die Wirtschaftspläne ausgerichtet werden; durch Handeln nach dem wirtschaftlichen Prinzip wird die Auswahl der Mittel bestimmt, um die Zwecke zu erreichen. »Egoismus« und

$38 \quad \mathrm{Zu}$ den wenigen Ausnahmen gehören beispielsweise Schlecht (2000) sowie Schumann und Nutzinger (2009).

$39 \quad$ Eucken (1952/1990: 352).

$40 \quad$ Vgl. Eucken (1952/1990: 352). Eucken zitiert hier wörtlich - allerdings ohne Quellenangabe Kant (1798/1900ff.: 130 [Anth AA VII, 130]). Offenbar setzt er die genaue Kenntnis von Kants Egoismus-Definition als Bildungshintergrund voraus. 
»wirtschaftliches Prinzip« befinden sich also gleichsam auf verschiedenen Ebenen. ${ }^{" 41}$

Seine kategorialen Differenzierungen fortführend, unterscheidet Eucken dann weiter zwischen einer einzelwirtschaftlichen und einer gesamtwirtschaftlichen Ausprägung des wirtschaftlichen Prinzips. Er entwickelt diese Unterscheidung anhand des folgenden Beispiels:

„In der Zeit zwischen 1945 und 1948 war es geradezu ein Kennzeichen der deutschen Wirtschaft, dass Einzelinteresse und wirtschaftliches Gesamtinteresse weit auseinanderklafften. War es die Schuld der Tausende von Menschen, die weite Strecken mit der Bahn zurücklegten, um Schuhe, Textilwaren, Bügeleisen usw. für Kartoffeln einzutauschen? Ohne Zweifel handelten sie nach dem wirtschaftlichen Prinzip. Handelten sie »egoistisch«, wenn sie etwa als Familienväter bemüht waren, für ihren Haushalt Lebensmittel zu besorgen? Jedenfalls entsprach ihre Arbeit - vom Gesamtprozess aus gesehen - keineswegs dem wirtschaftlichen Prinzip. [...] Das wirtschaftliche Prinzip war unter den gegebenen Umständen in seiner individuellen und allgemeinen Anwendung nicht zur Deckung zu bringen. “42

Dritter Schritt: Gestützt auf seine kategorialen Unterscheidungen, kann Eucken die Frontstellung der Ausgangspositionen konstruktiv kritisieren - und überwinden. Im Hinblick auf seine konstruktiv(istisch)e Kritik liest man:

„Die Antithese Eigennutz und Gemeinwohl verdeckt mehr das Problem, das hier Tag für Tag zu bewältigen ist, als dass sie es beschreibt. [...] Die eine Grundansicht leugnet die Harmonie zwischen dem Handeln der einzelnen Menschen nach dem wirtschaftlichen Prinzip; die andere behauptet sie.

Beide irren. Beide glauben an eine gegebene Konstruktion der Welt in dem von ihnen behaupteten Sinne.

Nun aber zeigte die Analyse der Tatbestände, dass es von den jeweils realisierten Ordnungsformen abhängt, ob die Herstellung der Harmonie gelingt oder nicht. Wir haben also nicht Harmonie oder Disharmonie als wirtschaftspolitisches Datum hinzunehmen. Vielmehr besteht die wesentliche Aufgabe darin, Ordnungsformen zu realisieren, in deren Rahmen sich eine Harmonie einstellt." ${ }^{\text {"3 }}$

Eucken hält die Frontstellung der Ausgangspositionen für verfehlt. Deshalb bezieht sich seine konstruktiv(istisch)e Kritik auch auf die intellektuelle Fehlorientierung, die man sich einhandelt, wenn man innerhalb des Denkhorizonts dieser Frontstellung haften bleibt. Hierzu liest man:

„Üblich ist es, die Aufgabe, die mit der Spannung zwischen Einzelinteressen und Gesamtinteresse gegeben ist, ausschließlich als ein Problem der sittlichen Erziehung anzusehen. Man spricht von der Notwendigkeit, Eigennutz oder Gewinn-

\footnotetext{
41 Eucken (1952/1990: 352f., H. i. O.).

$42 \quad$ Eucken (1952/1990: 353).

43 Eucken (1952/1990: 354f., H. i. O.). In der zitierten Passage gibt es - hin zur Wortwahl Übereinstimmungen mit Kant (1795/1900ff.: 370 [ZeF AA VIII 370]).
} 
streben oder »kapitalistischen Geist« zu überwinden, und erhofft davon, dass nun alle Menschen dem Gesamtinteresse dienen." 44

Eucken weist diese Vorstellung nicht nur als verfehlt zurück. Er überbietet sie argumentativ, indem er zeigt, dass sich das Problem auf anderem Wege weitaus besser lösen lässt. Hierzu liest man:

„Mit Schelten gegen Eigennutz, kapitalistische Gewinnsucht u. dgl. wird das Problem, wie Einzelinteresse und Gesamtinteresse in Harmonie gebracht werden, nicht gefördert.“45 „Von den Menschen darf nicht gefordert werden, was allein die Wirtschaftsordnung leisten kann: ein harmonisches Verhältnis zwischen Einzelinteresse und Gesamtinteresse herzustellen." “46

Blickt man auf diese insgesamt dreischrittige Gedankenführung aus einer ordonomischen Perspektive, so gewinnt man folgendes Bild von Euckens ordnungsethischer Argumentation: Im ersten Schritt zeichnet Eucken nach, wie ein Gegensatz konstruiert wird. Im zweiten Schritt re-konstruiert er die Problemstellung, um im dritten Schritt den Gegensatz als vermeintlichen Gegensatz zu de-konstruieren - und zu überwinden. Diese Überwindung lässt sich mit Hilfe von Abbildung 3 als orthogonale Positionierung interpretieren: als Überwindung der Wahrnehmung eines Tradeoffs durch einen Wechsel der Denkrichtung um 90.

Die Pointe dieser orthogonalen Positionierung besteht darin, die im zeitgenössischen Moraldiskurs geforderte Aufopferung des Eigeninteresses mit dem Überbietungsargument zu kritisieren, dass ein solches Opfer weder notwendig noch hinreichend ist, um das angestrebte Gemeinwohl zu verwirklichen. Mit dieser Pointe verbinden sich zwei Botschaften, die sich graphisch sehr anschaulich nachvollziehen lassen.

\footnotetext{
$44 \quad$ Eucken (1952/1990: 366).

$45 \quad$ Eucken (1952/1990: 358).
}

$46 \quad$ Eucken (1952/1990: 368). Diese Aussage ist nicht dahingehend misszuverstehen, dass Eucken etwas gegen sittliche Erziehung hätte. Das Gegenteil ist der Fall. So vertritt beispielsweise Eucken (1952/1990: 368) die Auffassung, „dass das Problem der Spannung zwischen Einzelinteresse und Gesamtinteresse durch sittliche Erziehung erleichtert, aber nicht gelöst werden kann. Sie kann z.B. wesentliche Mängel zentraler Wirtschaftslenkung nicht beseitigen. Umgekehrt wird die Bewältigung des Ordnungsproblems [...] durch sittliche Erziehung erleichtert, wobei aber diese Erziehung zugleich auf die Ordnungspolitik gerichtet sein sollte." Eucken bleibt also auch in dieser Hinsicht seinem Programm wissenschaftlich instruierter Aufklärung treu. Besonders deutlich wird dies bei Eucken (1938, 1947): Hier zielt ökonomische Bildung nicht darauf ab, dass sich der Einzelne im Wirtschaftsprozess anders verhalten möge, sondern vielmehr darauf, bei den Bürgern die Urteilskraft auszubilden, dass eine gesellschaftlich erwünschte Neuausrichtung einzelner Wirtschaftsprozesse einer institutionellen Gestaltung des Ordnungsrahmens bedarf. 


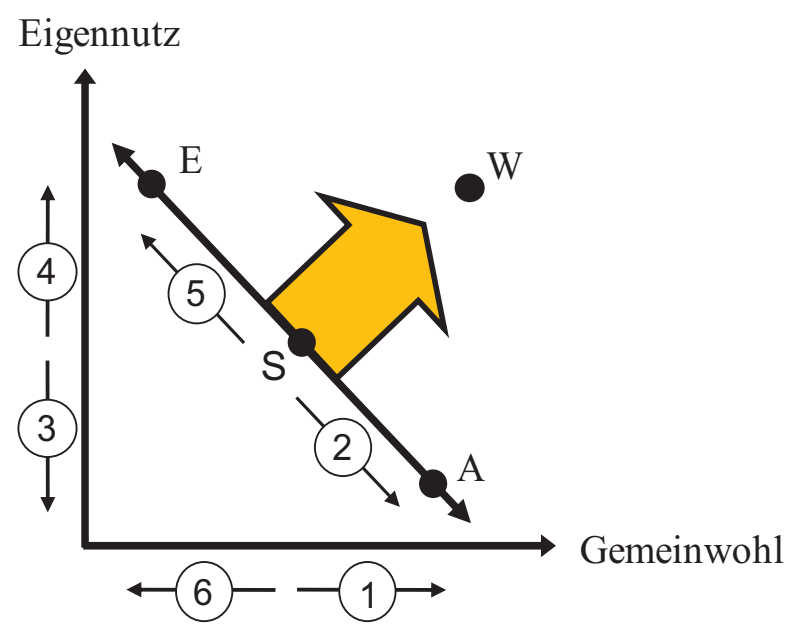

Abbildung 3: Euckens orthogonale Positionierung

Erstens: Fasst man das Spannungsfeld zwischen Eigennutz und Gemeinwohl als Trade-off, dann scheint es zunächst so, als werde das relevante Spektrum von den beiden Polen des Egoismus (E) und Altruismus (A) begrenzt. Innerhalb des Denkhorizonts dieser Frontstellung (Pfeil 2) ist es unausweichlich, um des Gemeinwohl willens (Pfeil 1) moralisch vom einzelnen Individuum zu fordern, auf sein eigenes Interesse zu verzichten (Pfeil 3). Und umgekehrt ist es innerhalb des Denkhorizonts dieser Frontstellung (Pfeil 5) unausweichlich, die Verfolgung des eigenen Interesses (Pfeil 4) als Beeinträchtigung des Gemeinwohls (Pfeil 6) aufzufassen und deshalb als unmoralisch zu verurteilen.

Zweitens: Erweitert man den Denkhorizont und rückt die ordnungspolitische Möglichkeit ins Blickfeld, den Widerstreit zwischen Eigennutz und Gemeinwohl nicht auf der Ebene der Handlungsgesinnungen, sondern auf der Ebene der institutionellen Handlungsbedingungen und ihrer Anreizwirkungen aufzulösen, dann lässt sich - repräsentiert durch den Pfeil in nord-östlicher Richtung - eine neue Denkrichtung identifizieren, die die Frontstellung der Ausgangspositionen überwindet und eine völlig neue Verhältnisbestimmung ermöglicht: Anstatt um des Gemeinwohls willen moralisch zu fordern, Abstriche beim Eigeninteresse zu machen, kann das Gemeinwohl nun moralisch gefördert werden (Pfeil 1), indem das Eigeninteresse ordnungspolitisch für das Gemeinwohl in Dienst genommen wird (Pfeil 4). In moderner Diktion entspricht dies einer Umstellung von Win-Lose auf Win-Win (W). Bei Eucken liest man hierzu explizit:

„Den spontanen Kräften der Menschen zur Entfaltung zu verhelfen und zugleich dafür zu sorgen, dass sie sich nicht gegen das Gesamtinteresse wenden, ist das Ziel, auf das sich die Politik der Wettbewerbsordnung richtet. - Man hat behauptet, die Wettbewerbsordnung appelliere ausschließlich an den Eigennutz oder an die Selbstsucht als Triebkraft. Das ist nicht wahr. Sie ist realistisch genug, um 
die ungeheure Kraft des Egoismus und des Selbsterhaltungstriebs in Rechnung zu stellen, aber sie weiß zugleich, dass andere Motive im Einzelinteresse der Haushalte und Betriebe ebenfalls zum Ausdruck kommen. Noch mehr: Sie ist der einzige Ordnungstyp, welcher die Kräfte des Egoismus bändigt. Im zentralverwaltungswirtschaftlichen Typ entfalten sich Egoismus und Willkür der Führerschicht und ihrer Bürokratie [...] Die Wettbewerbsordnung aber zwingt auch den reinen Egoisten, für das Gesamtinteresse tätig zu sein; z.B. einen Kaufmann oder Unternehmer, der rein egoistisch handelt, sinnvoll (und ohne es zu wollen) der Überwindung der Knappheit an Konsumgütern zu dienen." ${ }^{47}$ „Allein in der Wettbewerbsordnung würde - gleichsam durch List - die Vielfalt der egoistischen Einzelinteressen nicht gegeneinander arbeiten, sondern in Harmonie miteinander wirken. “48

(2) Wie denkt Eucken das Verhältnis zwischen Ordnungsethik und Ordnungspolitik? Reklamiert er - als Ökonom - einen Primat der Wirtschaft vor der Moral? Einzelne Formulierungen könnten in diese Richtung weisen, etwa wenn Eucken (1952, 1990; S. 323) schreibt: „Es kommt alles darauf an, dass der Ordnungsgedanke, der Gedanke der freien Ordnung, auch in die soziale Gesinnung mit aufgenommen wird. “ Aber dieser Schein trügt. - In Euckens Schriften findet sich eine klar abschlägige Antwort auf die hier aufgeworfene Frage nach einem Primat der Wirtschaft. Diese Antwort lässt sich hier in drei Schritten gedanklich nachvollziehen.

Erster Schritt: Eucken diskutiert explizit, warum er der Frage der Wirtschaftslenkung einen so hohen Stellenwert einräumt. Hierzu liest man:

„Ein ganz konkretes unabdingbares Problem des Alltags ist zu lösen: Die Überwindung der wirtschaftlichen Knappheit. Von der Lösung dieses Problems hängt die Lösung vieler anderer Probleme ab. Der sachliche Vorrang, den die Wettbewerbsordnung der wirtschaftlichen Notwendigkeit einräumt, hat mit materialistischen Auffassungen nichts zu tun. Hier ist keine Wahl: Wenn die ökonomische Notwendigkeit als untergeordnet vernachlässigt wird [...], dann werden die Menschen nur noch in einem viel höheren Grade von der Wirtschaft abhängig. Dann ist die Freiheit wirklich in Gefahr. “49

Es ist also nicht der materielle Reichtum, der für Eucken im Vordergrund steht, wenn er mit seinen Schriften für eine Wettbewerbsordnung plädiert, sondern vielmehr die historische Erfahrung, dass eine dysfunktionale Wirtschaftslenkung - aufgrund einer Interdependenz der Ordnungen von Wirtschaft, Recht, Staat und Gesellschaft - zu totalitären Freiheitsbedrohungen geführt hat. Dies ist eine Erfahrung, von der Eucken annimmt, dass sie kein Bürger wiederholen möchte. Vor diesem Hintergrund lautet sein ordnungspolitisches Argument: „Wenn man die Konsequenzen des Kollektivismus nicht will, dann muss man wollen, dass das Gesetz des Wettbewerbs herrscht. “50

\footnotetext{
$47 \quad$ Eucken (1952/1990: 365).

$48 \quad$ Eucken (1952/1990: 367).

$49 \quad$ Eucken $(1952 / 1990: 370)$.

$50 \quad$ Eucken (1952/1990: 371).
} 
Zweiter Schritt: Eucken diskutiert explizit, inwiefern die Frage der Wirtschaftslenkung und die Frage der Moral - ähnlich wie zwei Seiten derselben Medaille - eng und sogar systematisch zusammengehören. Hierzu liest man:

\begin{abstract}
„Dass der Totalitarismus ein Programm hat, wissen die Menschen. Dass es demgegenüber ein Programm der Freiheit gibt - die Wettbewerbsordnung wissen sie nicht oder nicht genug. Das ökonomisch-technische Problem, das in der Wettbewerbsordnung zu lösen versucht wird, wird nicht gesehen oder doch in seiner Bedeutung auch für andere Lebensgebiete unterschätzt. Es ist aber nur die eine Seite der Wettbewerbsordnung, dass sie auf die Durchsetzung der ökonomischen Sachgesetzlichkeit dringt. Ihre andere Seite besteht darin, dass hier gleichzeitig ein soziales und ethisches Ordnungswollen verwirklicht werden soll. Und in dieser Verbindung liegt ihre besondere Stärke. Denn ein sozial-ethisches Wollen ohne Verbindung mit der ökonomischen Sachlogik ist ebenso ohnmächtig, wie andererseits die wirtschaftliche Sachlogik nicht zur Auswirkung kommt, wenn nicht ein soziales Ordnungswollen die Gestaltung der Formen beeinflusst. “51
\end{abstract}

Eucken zieht also eine enge Verbindung zwischen dem ordnungspolitischen Aspekt einer preislichen Verhaltenskoordination über Wettbewerbsmärkte und dem ordnungsethischen Aspekt eines moralischen - in letzter Konsequenz freiheitsmotivierten - Ordnungswollens. Und sodann weist er diese enge Verbindung als die eigentliche Stärke der Wettbewerbsordnung aus, die für ihn ein Programm der Freiheit ist, weil sie sich an der Idee der Menschenwürde orientiert und diese Idee gesellschaftspolitisch zu implementieren hilft.

Dritter Schritt: Eucken war darauf bedacht, seine Theoriekonzeption darauf auszurichten, den öffentlichen Diskurs durch Argumente zu bereichern, die einen Weg aufzeigen, wie - nicht moralische Intuitionen, wohl aber - moralische Intentionen auch unter den Bedingungen einer wettbewerblich verfassten Marktwirtschaft - und stärker noch: entgegen dem oberflächlichen Eindruck sogar durch diese Bedingungen - zur Geltung kommen können. ${ }^{52} \mathrm{Ihm}$ kam es darauf an, moralische Anliegen nicht mit einem Verweis auf den wirtschaftlichen Sachzwang zurückzuweisen, sondern sie ordnungspolitisch verwirklichen zu helfen, und zwar mit Hilfe eines ökonomisch instruierten Ebenenwechsels von den Spielzügen zu den Spielregeln. Insofern lässt sich Eucken zumindest implizit die ordnungsethische Kernthese zuschreiben, dass in Wettbewerbskontexten die institutionellen Rahmenbedingungen zum systematischen Ort der Moral avancieren. ${ }^{53}$ Explizit liest man bei ihm hierzu:

$51 \quad$ Eucken (1952/1990: 370f., im Original teilweise hervorgehoben). Bei Karl Homann (2002: 263) liest man in ganz ähnlicher Weise: „Ethik ohne Ökonomik ist leer, Ökonomik ohne Ethik ist blind.“

Bereits Eucken (1932: 321) hält es für einen ,ungemein weit verbreitete[n] Fehler, unerwünschte Folgen des Interventionismus nicht als solche zu erkennen, sondern die Ursachen im freien Spiel der Kräfte zu suchen“. In moderner Diktion ausgedrückt, macht Eucken kritisch darauf aufmerksam, dass in der Öffentlichkeit eine Tendenz besteht, Fälle von Politikversagen bei der Ordnungsgestaltung fälschlicherweise als Marktversagen zu interpretieren, also den eigentlichen Missstand nicht den Spielregeln, sondern den Spielzügen zuzurechnen.

Zu dieser erstmals von Karl Homann formulierten These vgl. ausführlich Pies (2010). 


\begin{abstract}
„Man sagt sehr oft und mit Recht, die Lösung der sozialen Fragen sei eine Frage der sozialen Moral; die Gesinnung der Menschen zueinander sei entscheidend [...] Das alles ist richtig. Aber es genügt nicht, dies nur einfach zu sagen und darüber das Ordnungsproblem zu vernachlässigen. Es kommt vielmehr darauf an, dass sich diese Gesinnung auch in der Gestaltung der Ordnung äußert." ${ }^{\text {"54 }}$
\end{abstract}

Blickt man auf diese insgesamt dreischrittige Gedankenführung aus einer ordonomischen Perspektive, so gewinnt man folgendes Bild von Euckens Argumentation: Im ersten Schritt lehnt Eucken einen Primat der Wirtschaft vor der Moral ausdrücklich ab. Im zweiten Schritt macht er geltend, dass Wirtschaftslenkung und Moral wie zwei Seiten einer Medaille zusammengehören, um im dritten Schritt vor Augen zu führen, dass es ihm letztlich um einen Primat der Moral geht, weil die funktionale Wirtschaftslenkung dafür in Dienst genommen werden soll, den Menschen die Option auf ein möglichst selbstbestimmtes Leben zu eröffnen. Aus Euckens Sicht ist es eben ein ethisch fundiertes Wollen, das ordnungspolitisch zur Geltung kommen soll. Und genau das macht ihn zu einem Klassiker der Ordnungsethik.

\title{
5. Zur Aktualisierung der ordnungspolitischen Konzeption: eine ordonomische Perspektive
}

„Die Wissenschaft ist stets beides zugleich: sie ist revolutionär und traditionell. Revolutionär - insofern sie radikal fragt und fragen muss; traditionell - weil sie nicht Fragestellungen und Problemlösungen von Männern über Bord werfen darf, die sehr Gewichtiges zu sagen hatten. “55

Untersucht man Walter Euckens Gesamtwerk und die hier entwickelte Konzeption eines Denkens in Ordnungen aus dem Blickwinkel des ordonomischen Forschungsprogramms, dann treten deutlich mehrere Stärken hervor, an die eine moderne Theoriebildung anknüpfen kann. Zugleich werden aber auch einige Schwächen deutlich, die eine moderne Theoriebildung vermeiden muss, wenn sie dem Anspruch gerecht werden will, das klassische Erbe der Euckenschen Ordnungspolitik - und nota bene: das klassische Erbe der Euckenschen Ordnungsethik - konstruktiv(istisch) fortzuentwickeln und für aktuelle Problemlösungen fruchtbar zu machen. Insgesamt sind es drei Stärken und drei Schwächen, auf die hier aufmerksam gemacht werden soll.

(1) Zuerst zu den Stärken: Walter Eucken beschäftigt sich in einer für Ökonomen sowohl quantitativ wie auch qualitativ - ganz außergewöhnlichen Art und Weise nicht nur mit Fragen der Sozialstruktur, sondern auch mit Fragen der Semantik. Ihm geht es nicht nur um eine möglichst modellgestützte Analyse institutioneller Arrangements und ihrer Anreizwirkungen, sondern insbesondere auch um eine ideologiekritische Analyse der für die intellektuelle Auseinandersetzung zentralen Begriffe und der ihnen zugrunde liegenden Denkkategorien, wobei ihm gerade hier sein philosophischer Bildungshintergrund sehr zugute kommt. Dies ist die erste Stärke.

Die zweite Stärke knüpft unmittelbar daran an. Als Ökonom interessiert sich Eucken für institutionelle Reformen zur Verbesserung der Wirtschaftslenkung. Hier hat er -

\footnotetext{
$54 \quad$ Eucken (1952/1990: 323).

$55 \quad$ Eucken $(1940 / 1959:$ X).
} 
vermittelt durch die von ihm identifizierte Interdependenz der Ordnungen - ein letztlich gesellschaftspolitisches Steuerungsproblem vor Augen. Aber gerade als Ökonom kann Eucken nicht umhin, ein Aufklärungsproblem zu identifizieren, das der unmittelbaren Lösung des Steuerungsproblems im Wege steht. Deshalb sucht er nach einem Weg, aus der Ökonomik heraus einer Lösung sowohl des Steuerungsproblems und des diesem vorausliegenden Aufklärungsproblems zuzuarbeiten. Genau darauf ist seine ordnungspolitische Konzeption berechnet, indem sie darauf abzielt, wissenschaftliche Beiträge zu generieren, die das Denken zur ordnenden Potenz erheben. Als Methode demokratischer Politikberatung ist dies vorbildlich.

Eine dritte Stärke zeigt sich darin, wie Eucken es vermeidet, seine ordnungspolitische Stellungnahme auf einen wertstrittigen Dezisionismus zu gründen: Er war konfrontiert mit einer zeitgenössischen Auseinandersetzung zwischen Liberalismus und Sozialismus, in der die eine Seite sich auf den Wert der Freiheit berief, während die andere Seite die Werte sozialer Sicherheit und sozialer Gerechtigkeit für sich reklamierte. Eine besondere Leistung Euckens bestand nun darin, diese Auseinandersetzung nicht zum Nennwert zu nehmen und sich dann - als Liberaler - einfach auf die Seite der Freiheit zu schlagen. Stattdessen rekonstruiert und dekonstruiert er diese Frontstellung, um sie zu überwinden. Dabei entsteht etwas Neues: Euckens Kritik am Sozialismus korrespondiert eine Kritik am Laissez-faire-Liberalismus, den er als Altliberalismus einstuft und in zweierlei Hinsicht durch seinen neuen Ordo-Liberalismus überbietet: zum einen im Hinblick auf die Fundierung liberaler Prinzipien, die er nicht naturrechtlich begründet, sondern - ökonomisch instruiert - als öffentlich nachvollziehbare Zweckmäßigkeitsargumente ausweist; zum anderen im Hinblick auf die Frage der Staatstätigkeit, wobei Eucken staatsminimalistischen Vorstellungen eine klare Absage erteilt und mit seiner Konzeption von Ordnungspolitik zugleich eine neue Differenzierung einführt, auf welcher Ebene der Staat aktiv werden sollte und auf welcher Ebene besser nicht.

(2) Dass ein Autor, der vor nunmehr über 60 Jahren unerwartet früh aus dem Leben gerissen wurde und ausgerechnet sein ordnungspolitisches Hauptwerk in einem teilweise fragmentarischen Zustand hinterlassen hat, in zahlreichen Detailfragen von der aktuellen Forschung überholt worden ist, versteht sich von selbst und bedarf hier keiner weiteren Ausführungen. Aber gerade im Hinblick auf die Theoriearchitektonik des Gesamtwerks zeigen sich einige Schwachpunkte, deren man sich bewusst sein sollte, wenn man Euckens Analysetradition mit Aussicht auf Erfolg fortentwickeln möchte.

Der erste Schwachpunkt besteht darin, dass Euckens ordnungspolitische Konzeption mit einer Typologie arbeitet, die zwei Arten der Wirtschaftsplanung unterscheidet. Dass Eucken mit Hilfe dieser Typologie eine enorme Komplexitätsreduktion gelingt, die es ihm erlaubt, sehr weitreichende Aussagen über die absehbaren Konsequenzen alternativer Gesamtentscheidungen zu treffen, ist zunächst eine Stärke seines Ansatzes. Diese Stärke mutiert aber unter der Hand zu einer Schwäche, sobald es nicht mehr darum geht, auf der konstitutionellen Ebene eine Entscheidung über die Wirtschaftsverfassung herbeizuführen, indem man sich entweder für eine Wettbewerbsordnung oder für eine Zentralplanung entscheidet. Sobald man sich innerbalb einer Marktwirtschaft - und insofern auf der sub-konstitutionellen Ebene - mit konkreten 
Politikalternativen konfrontiert sieht, verliert die Planungstypologie ihr heuristisches Potential. Der ordnungspolitische Ansatz läuft dann sogar Gefahr, selbst ideologisch missbraucht werden zu können, weil die in Anspruch genommene Annahme, es gebe letztlich nur zwei Alternativen, immer dazu verleiten kann, Abweichungen von der (vermeintlich) reinen Lehre als erste Schritte auf dem Weg in den Kollektivismus zu brandmarken. Einer konstruktiven Diskussion gesellschaftspolitischer Gestaltungsoptionen ist ein solcher Alternativenradikalismus nicht wirklich förderlich, weil er letztlich dazu tendiert, in wertstrittige Auseinandersetzungen hineinzuführen - bzw. zurückzufallen -, anstatt diese wissenschaftlich überwinden zu helfen. ${ }^{56}$

Der zweite Schwachpunkt betrifft die Konzeptualisierung des Verhältnisses von Wirtschaft und Staat. Dass Eucken hier eine Interdependenz der Ordnungen identifiziert, ist zunächst eine Stärke seines Ansatzes. Sie lässt Eucken - nicht dem Begriff, wohl aber der Sache nach - zu einem Pionier der Theorie (und theoretischen Kritik) des Rent-Seeking werden. Diese Stärke mutiert aber unter der Hand zu einer Schwäche, sobald es darum geht, den Beitrag zu bestimmen, den Unternehmen - und allgemein: Organisationen, vor allem auch Organisationen der Zivilgesellschaft - für die Gesellschaft zu leisten vermögen. Es ist ein Defizit der Theoriebildung, wenn Eucken Unternehmen nur als Regelnehmer und ausschließlich den Staat als Regelgeber aufgefasst wissen will. Zwar hat Eucken gute Gründe, gegenüber der Privilegiensuche und dem Machtstreben wirtschaftlicher Akteure hochgradig skeptisch und stets auf der Hut zu sein. Dennoch unterschätzt er das Potential gesellschaftlicher Lernprozesse, das darin liegt, dass Unternehmen als Akteure im System der Wettbewerbsmärkte nicht nur destruktive, sondern auch ausgesprochen konstruktive Rückwirkungen auf dieses System ausüben können. Letztlich ist dies eine Frage der Rahmenordnung für politische Aktivitäten und hängt beispielsweise davon ab, wie der wettbewerbliche Zugang zur politischen Willensbildung geregelt ist, welche Transparenzvorschriften zu beachten sind, wo die Grenze zwischen legitimer und illegitimer Beeinflussung (z.B. in Form von Korruption) gezogen wird und wie hart das Überschreiten dieser Grenze geahndet wird. Insofern ist es hier möglich - und nötig! -, mit Eucken gegen Eucken zu denken: Gerade aufgrund einer Interdependenz der Ordnungen sollte man nicht ausschließen, sondern vielmehr institutionell dafür Sorge tragen, dass Unternehmen als Corporate Citizens sich - gemeinsam mit zivilgesellschaftlichen Organisationen - in der politischen Arena als ordnende Potenzen betätigen können. ${ }^{57}$

Euckens ordnungspolitische Konzeption ist auf ein einziges - historisch singuläres - Problem zugeschnitten. Sie soll dazu beitragen, dass nach einem halben Jahrhundert verfehlter Experimente in Deutschland und darüber hinaus endlich eine Wirtschafts- und Gesellschaftsordnung etabliert wird, die nicht wieder totalitäre Tendenzen auslöst. Hierbei gelingt Eucken eine orthogonale Positionierung zur wertstrittigen Auseinandersetzung zwischen Liberalismus und Sozialismus. - Um orthogonale Positionierungen jedoch zur Methode zu erheben, muss man vom Denken in Ordnungen zu einem Denken in Anreizen übergehen. Deshalb setzt das ordonomische Forschungsprogramm darauf, Euckens Ordnungstypologie durch das wesentlich leistungsfähigere Schema einseitiger und mehrseitiger Dilemmastrukturen zu ersetzen: Das soziale Dilemma modelliert die sozialstrukturelle Grundlage für semantische Aufklärungsbeiträge in Form orthogonaler Positionierungen. Vgl. hierzu ausführlich Pies (2009a).

57 Man kann diesen Sachverhalt auch so ausdrücken, dass Euckens Ordnungsethik primär wirtschaftsethisch angelegt ist und dass man bei ihm allenfalls indirekte Anregungen findet, wie 
Eine dritte Schwäche kommt hinzu. Eucken unterschätzt aufgrund seines generellen Kartellverdachts nicht nur, dass Unternehmen durchaus auch gemeinwohlorientiert an der politischen Rahmensetzung mitarbeiten können. Er unterschätzt aufgrund seines generellen Kartellverdachts zudem, dass das von der Wirtschaft geschaffene Recht insbesondere die vertraglichen Regelungen eines Unternehmens mit seinen Mitarbeitern und Kunden sowie Lieferanten - nicht unbedingt wettbewerbsbeschränkende Wirkungen entfalten muss, sondern als Instrument zur Lösung von Interaktionsproblemen durchaus auch zur besseren Funktionsweise des marktwirtschaftlichen Systems beitragen kann. Dass Unternehmen sich als Regelgeber innerhalb der wirtschaftlichen Arena betätigen, ist ein elementares Kernelement ihrer Wertschöpfung. Dies aber hat Eucken gar nicht im Blick. Deshalb ist auch sein Verständnis von Ordnungspolitik aus heutiger Sicht zu undifferenziert und gewissermaßen zu sta(a)tisch'. Das meint zweierlei. Einerseits unterschätzt Eucken die Dynamik gesellschaftlicher Lernprozesse zur (Er-)Findung funktionaler Anreiz-Arrangements, die innerhalb des politisch verabschiedeten Gesetzesrahmens stattfindet, indem Unternehmen gemeinsam mit ihren Interaktionspartnern mit Problemlösungen experimentieren, um immer neue Wertschöpfungspotentiale auszureizen. Andererseits überschätzt Eucken die Möglichkeiten des Staates, all jene Rahmenbedingungen setzen zu können, die für ein reibungsloses Funktionieren der Wirtschaft erforderlich sind. Hier mag man zum einen an das auf globalisierten Märkten zunehmend akute Problem internationaler Regelsetzung denken. Zum anderen aber, und darauf soll hier besonders hingewiesen werden, ist daran zu denken, dass der Staat - gemeinsam mit anderen Akteuren, namentlich mit zivilgesellschaftlichen Organisationen und unter Begleitung einer kritischen Berichterstattung sowie öffentlichen Meinungsbildung - die Aufgabe wahrzunehmen hat, für ein ,enabling environment ${ }^{\star}$ zu sorgen, das dem Regelsetzungsverhalten der Unternehmen Raum zur Entfaltung bietet und es zugleich so ausrichtet, dass hier im Sinne des Gemeinwohls Beiträge zur Lösung gesellschaftlicher Probleme geleistet werden. Man kann diese Herausforderung mit dem Begriff einer „Ordnungspolitik zweiter Ordnung“ belegen: Während es einer Ordnungspolitik erster Ordnung darum geht, Spielregeln festzulegen, die den Spielzügen einen verbindlichen Rahmen geben, setzt eine Ordnungspolitik zweiter Ordnung Anreize zur Anreizsetzung in und durch Unternehmen. Sie steuert Spielzüge, die ihrerseits in einem Regelsetzungsverhalten bestehen. ${ }^{58}$

sich - mit Eucken gegen Eucken denkend - eine ordnungsethische Unternehmensethik entwickeln ließe. Das ordonomische Forschungsprogramm unterbreitet genau hierfür ein Theorieangebot. Es ist um die Idee zentriert, dass Unternehmen als Wertschöpfungsagenten im gesellschaftlichen Auftrag ,Moral als Produktionsfaktor ${ }^{6}$ einsetzen können und dass sich just diese Wertschöpfungslogik, die auf wechselseitige Vorteilsgewährung abzielt, durch Unternehmen - in Form von Corporate Citizenship - von der wirtschaftlichen auch in die politische Arena übertragen lässt. Vgl. hierzu Pies (2009b) sowie Pies et al. (2009a) und (2009b). Vgl. ferner Hielscher et al. (2009) sowie Pies et al. (2011).

Am Beispiel: Zur wirksamen Bekämpfung von Korruption ist ein gesetzliches Verbot zwar notwendig, aber nicht hinreichend. Hinzu kommen müssen Anreize für Unternehmen, damit diese organisationsintern geeignete Vorkehrungen treffen, um durch sanktionsbewehrte Regeln die erforderlichen Präventions-Anreize an ihre Mitarbeiter weiterzugeben. Vgl. hierzu ausführlich Pies (2008). Ein weiteres Beispiel betrifft die Regelung von Haftungsbeschränkungen innerhalb 


\section{Fazit}

Vor diesem Hintergrund lässt sich abschließend festhalten: Gerade weil es möglich ist, mit Eucken gegen Eucken zu denken, gebührt ihm der Status eines Klassikers. Diesen Klassikerstatus hat Eucken aber nicht nur für seine Ordnungspolitik verdient, sondern auch für seine Ordnungsethik. In der Tat hängt bei ihm beides eng zusammen: Einerseits ist die Ordnungspolitik funktional für die Ordnungsethik, weil sie institutionell in genau jenen Wettbewerbskontexten für eine Kompatibilität von Eigennutzstreben und Gemeinwohlorientierung sorgen kann, in denen der einzelne Wirtschaftsakteur moralisch überfordert wäre, diese gesellschaftlich wünschenswerte Kompatibilität allein auf sich gestellt herbeizuführen. Andererseits ist die Ordnungsethik funktional für die Ordnungspolitik, weil sie es vermag, in der öffentlichen Moralkommunikation auftretende Kategorienfehler kritisch identifizieren und konstruktiv korrigieren zu helfen, die in Form von Denkblockaden der institutionellen Gestaltung funktionaler Anreizarrangements als Hindernisse im Wege stehen. Eucken selbst hat dieses fruchtbare Feld zwischen Ordnungsethik und Ordnungspolitik in beiderlei Richtung produktiv bearbeitet. Deshalb lohnt es sich, Walter Eucken als einen Klassiker der Ordnungsethik zu lesen: als einen Autor, dessen Werk ein derzeit längst noch nicht ausgeschöpftes Potential bereithält, moderne Theoriebildungsanstrengungen im thematischen Umfeld der Wirtschaftsethik konzeptionell inspirieren zu können. Als These zugespitzt: Zum Klassiker avanciert Eucken nicht so sehr aufgrund des Inhalts als vielmehr aufgrund der Form seines Denkens, weniger aufgrund einzelner Argumente als vielmehr aufgrund seiner Art des Argumentierens, die vom Ansatz her - konstruktivistisch - darauf zugeschnitten ist, Theorie praktisch werden zu lassen, indem sie im Modus wissenschaftlicher Aufklärung dazu beiträgt, politische und moralische Denkblockaden aufzulösen, die ansonsten zu gravierenden Handlungsblockaden führen können.

\section{Literaturverzeichnis}

Buchanan, J. M./ Tullock, G. (1962/1990): The Calculus of Consent. Logical Foundations of Constitutional Democracy, Ann Arbor.

Dathe, U. (2009): Walter Euckens Weg zum Liberalismus (1914-1934), in: ORDO, Bd. 60, 5386.

Dathe, U./ Goldschmidt, N. (2003). Wie der Vater, so der Sohn? Neuere Erkenntnisse zu Walter Euckens Leben und Werk anhand des Nachlasses von Rudolf Eucken in Jena, in: ORDO, Bd. 54, 49-74.

Eucken, W. (1932): Staatliche Strukturwandlungen und die Krisis des Kapitalismus, in: Weltwirtschaftliches Archiv 36, 297-321.

Eucken, W. (1934/1954): Was leistet die Nationalökonomische Theorie?, in: ders.: Kapitaltheoretische Untersuchungen, 2. Aufl., Tübingen, 1-51.

Eucken, W. (1938/1947): Nationalökonomie wozu? 3. Aufl., Godesberg.

von Organisationen und die hiermit verbundenen Vergütungsstrukturen von Managern. Vgl. hierzu ausführlich Pies/Sass (2011). Zum Zusammenspiel von Unternehmen und staatlichen Akteuren sowie zivilgesellschaftlichen Organisationen im Rahmen von New-GovernanceProzessen vgl. Pies et al. (2010). Vgl. auch Pies/Hielscher (2008). 
Eucken, W. (1940/1959): Die Grundlagen der Nationalökonomie, 7. Aufl., Berlin u. a. O.

Eucken, W. (1948a): Das ordnungspolitische Problem, in: ORDO 1, 56-90.

Eucken, W. (1948b): Die soziale Frage, in: Synopsis, hrsg. von Edgar Salin, Basel, 111-131.

Eucken, W. (1949): Die Wettbewerbsordnung und ihre Verwirklichung, in: ORDO 2, 1-99.

Eucken, W. (1950): Vorwort des Herausgebers, in: Rudolf Eucken (1890/1950): Die Lebensanschauungen der großen Denker. Eine Entwicklungsgeschichte des Lebensproblems der Menschheit von Platon bis zur Gegenwart, hrsg. von Walter Eucken, 20. Auflage, Berlin, V-VII.

Eucken, W. (1951): Unser Zeitalter der Misserfolge. Fünf Vorträge zur Wirtschaftspolitik, Tübingen.

Eucken, W. (1952/1990): Grundsätze der Wirtschaftspolitik, hrsg. von Edith Eucken und K. Paul Hensel, 6. Aufl., Tübingen.

Grossekettler, H. (1997): Die Wirtschaftsordnung als Gestaltungsaufgabe. Entstehungsgeschichte und Entwicklungsperspektiven des Ordoliberalismus nach 50 Jahren Sozialer Marktwirtschaft, Münster.

Grossekettler, H. (2000): Ordnungstheorie und Recht. Die Rolle von Ökonomen und Juristen bei der Entwicklung und Verteidigung einer koordinationseffizienten Wirtschaftsverfassung, in: Leipold, H./ Pies, I. (Hrsg.): Ordnungstheorie und Ordnungspolitik. Konzeption und Entwicklungsperspektiven, Stuttgart, 429-453.

Grossekettler, H. (2003): Walter Eucken, Volkswirtschaftlicher Diskussionsbeitrag der Westfälischen Wilhelms-Universität Münster Nr. 347, Münster.

Hielscher, S./ Pies, I./ Beckmann, M. (2009): Wie können Corporate Citizens voneinander lernen? Ordonomische Anregungen für inter-organisationales Lernen im Global Compact der Vereinten Nationen, in: Zeitschrift für Wirtschafts- und Unternehmensethik (zfwu) 10(1), 41-65.

Homann, K. (1988): Rationalität und Demokratie, Tübingen.

Homann, K. (2002): Ökonomik: Fortsetzung der Ethik mit anderen Mitteln, in: ders.: Vorteile und Anreize, Tübingen, 243-266.

Homann, K./ Kirchner, C. (1995): Ordnungsethik, in: Jahrbuch für Neue Politische Ökonomie 14, 189-211.

Kant, I. (1795/1900ff.): Zum ewigen Frieden, in: Immanuel Kant: Gesammelte Schriften (Akademie Ausgabe), hrsg. von der Königlich Preußischen Akademie der Wissenschaften, Berlin, Band VIII, 341-386.

Kant, I. (1798/1900ff.): Anthropologie in pragmatischer Absicht, in: Immanuel Kant: Gesammelte Schriften (Akademie Ausgabe), hrsg. von der Königlich Preußischen Akademie der Wissenschaften, Berlin, Band VII, 117-333.

Pies, I. (2000): Ordnungspolitik in der Demokratie. Ein ökonomischer Ansatz diskursiver Politikberatung, Tübingen.

Pies, I. (2001): Eucken und von Hayek im Vergleich. Zur Aktualisierung der ordnungspolitischen Konzeption, Tübingen.

Pies, I. (2002): Theoretische Grundlagen demokratischer Wirtschafts- und Gesellschaftspolitik - Der Beitrag Walter Euckens, in: Pies, I./ Leschke, M. (Hrsg.): Walter Euckens Ordnungspolitik, Tübingen, 1-35.

Pies, I. (2008): Wie bekämpft man Korruption? Lektionen der Wirtschafts- und Unternehmensethik für eine, Ordnungspolitik zweiter Ordnung, Berlin.

Pies, I. (2009a): Moral als Heuristik. Ordonomische Schriften zur Wirtschaftsethik, Berlin. 
Pies, I. (2009b): Moral als Produktionsfaktor. Ordonomische Schriften zur Unternehmensethik, Berlin.

Pies, I. (2010): Karl Homanns Programm einer ökonomischen Ethik - „A View From Inside“ in zehn Thesen, Zeitschrift für Wirtschafts- und Unternehmensethik (zfwu) 11(3), 249261.

Pies, I./ Beckmann, M./ Hielscher, S. (2011): Competitive Markets, Corporate Firms, and New Governance - An Ordonomic Conceptualization, in: Pies, I./ Koslowski, P. (Hrsg.): Corporate Citizenship and New Governance - the Political Role of Corporations, Dordrecht u. a. O., 171-188.

Pies, I./ Hielscher, S. (2008): Der systematische Ort der Zivilgesellschaft. Welche Rolle weist eine ökonomische Theorie der Moral zivilgesellschaftlichen Organisationen in der modernen Gesellschaft zu? In: Zeitschrift für Wirtschafts- und Unternehmensethik (zfwu) 9(3), 337-344.

Pies, I./ Hielscher, S./ Beckmann, M. (2009a): Moral Commitments and the Societal Role of Business: An Ordonomic Approach to Corporate Citizenship, in: Business Ethics Quarterly 19(3), 375-401.

Pies, I./ Hielscher, S./ Beckmann, M. (2009b): Zum Curriculum der Wirtschafts- und Unternehmensethik - Anregungen für die Ausbildung von Führungskräften, in: Zeitschrift für Wirtschafts- und Unternehmensethik (zfwu) 10(2), 184-202.

Pies, I./ Sass, P. (2011): Wie sollte die Managementvergütung (nicht) reguliert werden? - Ordnungspolitische Überlegungen zur Haftungsbeschränkung von und in Organisationen, Diskussionspapier Nr. 2011-2 des Lehrstuhls für Wirtschaftsethik an der MartinLuther-Universität Halle-Wittenberg, Halle.

Pies, I./ von Winning, A./ Sardison, M./ Girlich, K. (2010): Sustainability in the Petroleum Industry: Theory and Practice of Voluntary Self-Commitments, Wirtschaftsethik-Studie Nr. 2010-1 des Lehrstuhls für Wirtschaftsethik an der Martin-Luther-Universität HalleWittenberg, Halle.

Rawls, J. (1971/1979): Eine Theorie der Gerechtigkeit. Übersetzt von Hermann Vetter, Frankfurt a. M.

Schlecht, O. (2000): Zur Ethik in Euckens Werk, in: Külp, B./ Vanberg, V. (Hrsg.): Freiheit und wettbewerbliche Ordnung, Freiburg u. a. O., 59-73.

Schumann, O. J./ Nutzinger, H. G. (2009): Ordoliberalismus und Gerechtigkeit: Zum Verhältnis von Eucken und Kant, MAGKS Diskussionspapier Nr. 48-2009.

Weber, M. (1904/1988): Die »Objektivität« sozialwissenschaftlicher und sozialpolitischer Erkenntnis, in: Gesammelte Aufsätze zur Wissenschaftslehre, hrsg. von Johannes Winckelmann, Tübingen, 146-214.

Weber, M. (1918/1988): Der Sinn der »Wertfreiheit« der soziologischen und ökonomischen Wissenschaften, in: ders.: Gesammelte Aufsätze zur Wissenschaftslehre, hrsg. von Johannes Winckelmann, Tübingen, 489-540. 